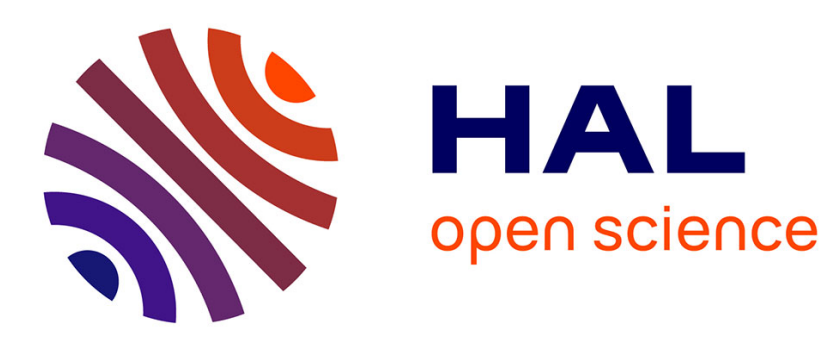

\title{
Interaction between interstitial-interstitial and interstitial-substitutional solute atoms in d-band metals
}

K. Masuda, T. Mori

\section{To cite this version:}

K. Masuda, T. Mori. Interaction between interstitial-interstitial and interstitial-substitutional solute atoms in d-band metals. Journal de Physique, 1976, 37 (5), pp.569-586. 10.1051/jphys:01976003705056900 . jpa-00208451

\section{HAL Id: jpa-00208451 https://hal.science/jpa-00208451}

Submitted on 1 Jan 1976

HAL is a multi-disciplinary open access archive for the deposit and dissemination of scientific research documents, whether they are published or not. The documents may come from teaching and research institutions in France or abroad, or from public or private research centers.
L'archive ouverte pluridisciplinaire HAL, est destinée au dépôt et à la diffusion de documents scientifiques de niveau recherche, publiés ou non, émanant des établissements d'enseignement et de recherche français ou étrangers, des laboratoires publics ou privés. 


\title{
INTERACTION BETWEEN INTERSTITIAL-INTERSTITIAL AND INTERSTITIAL-SUBSTITUTIONAL SOLUTE ATOMS IN d-BAND METALS
}

\author{
K. MASUDA and T. MORI \\ Department of Metallurgical Engineering \\ Tokyo Institute of Technology Ookayama, Meguro, Tokyo, Japan
}

(Reçu le 4 novembre 1975, accepté le 21 janvier 1976)

\begin{abstract}
Résumé. - On étudie, dans l'approximation des liaisons fortes, la contribution électronique à l'énergie d'interaction entre atomes interstitiels en solution dans les métaux de transition (interaction I-I). La méthode théorique utilisée dans ce travail est très semblable à celle qui a été développée par Einstein et Schrieffer pour les interactions d'adatomes. Cette méthode nous permet d'étudier l'atome de gaz dans les sites octaédriques comme dans les sites tétraédriques et nous permet également d'étudier l'interaction entre les atomes interstitiels et les atomes de substitution (interaction I-S). Des calculs ont été faits en fonction du remplissage de la bande, du niveau d'énergie de l'atome dissous, d'un élément de matrice $V_{0}$ du potentiel d'impureté sur le site de l'atome de substitution et d'un potentiel général de saut $V_{a}$ entre un atome interstitiel et les atomes plus proches voisins dans la matrice. Les résultats numériques soulignent généralement l'importance de la contribution électronique aux énergies d'interaction (interaction I-I et I-S). Avec des conditions raisonnables, on montre que notre modèle simple de l'interaction I-S est en bon accord avec les données expérimentales pour les alliages ternaires à base de fer contenant des atomes simples comme $\mathbf{N}$ ou $\mathrm{C}$. L'énergie d'interaction I-I varie souvent faiblement avec la position angulaire et on discute la possibilité d'une condensation interstitielle (transition de phase $\alpha-\alpha^{\prime}$ ) observée dans un système $\mathrm{Nb}-\mathrm{H}$. On compare également l'interaction soluté (I-I) estimée d'après la théorie élastique.
\end{abstract}

\begin{abstract}
The electronic contribution to the interaction energy between interstitial solute atoms (I-I interaction) in transition metals is investigated within the tight-binding approximation. The theoretical method used in this study closely parallels that developed by Einstein and Schrieffer for the adatom interaction. The method permits us to study the solution of the gas atom in the octahedral sites as well as in the tetrahedral sites and also allows us to investigate the interaction between the interstitial and substitutional solute atoms (I-S interaction). Calculations have been carried out as a function of band filling, solute atom energy level, an impurity potential matrix element $V_{0}$ on the substitutional atom site, and a general hopping potential $V_{a}$ between an interstitial atom and the nearest neighbour host atoms. The numerical results generally indicate the importance of the electronic contribution to the interaction energies (both I-I and I-S interaction). Under reasonable conditions, our simple model of the I-S interaction is shown to agree well with the experimental data for Fe-based ternary alloys containing the simple gas atoms such as $\mathrm{N}$ or $\mathrm{C}$. The angular position dependence of the I-I interaction energy is often weak and the possibility of the interstitial condensation $\left(\alpha-\alpha^{\prime}\right.$ phase transition) observed in a Nb-H system is discussed. Contact is also made with the solute (I-I) interaction based on the elastic theory.
\end{abstract}

1. Introduction. - Studies of the behaviour of interstitial solute atoms and the interaction between interstitial solute atoms in an interstitial solid solution (to be referred to as I-I interaction) have been of considerable theoretical and experimental interest in recent years. It is also of considerable concern to obtain information about the interaction between interstitial and substitutional solute atoms in an interstitial solid solution with substitutional atoms (to be referred to as I-S interaction). These interaction energies have been investigated by the measurements of the solute activity and the influence of solute changing of the half-width of the $\mathrm{X}$-ray reflection lines [1]. The elastic relaxation or internal friction methods are also used for this purpose [2]. On the other hand, these I-I or I-S interaction energies have been discussed theoretically by the elastic theory (including lattice statics) $[3,4]$ and other simple treatments such as quasichemical [5] or screened potential approaches [6]. The theory developed so 
far, however, neglects the covalent nature of the interstitial-host metal atom binding and probably could not be applied to the transition metal systems. The covalent nature induced by the electron hopping between the adsorbed atom and the host metal atoms is generally treated in the problem of chemisorption [7]. The adatom interaction due to the covalent nature in fact explains the surface structures observed, such as $c(2 \times 2),(2 \times 2)$ and $c(4 \times 2)$ [8]. In transition metal alloys, the covalency seems to play a significant role in some respects. For example, energies of solution of $\mathrm{H}$ atoms are much more negative in a number of transition metals and alloys than in normal metals (see Table I), pointing to a large role of the $\mathrm{d}$ band. In other light interstitial impurities $(B, C, N)$, most of their screening electrons seem to interact with the $\mathrm{d}$ band of the matrix. This is especially clear in recent measurements $[9,10]$ of hyperfine magnetic coupling of $\mathrm{Fe}$ by the Mössbauer effect, which gives a decrease in magnetic moments of $\mathrm{Fe}$ atoms near to $\mathrm{C}$ or N. On the other hand, an ultrahigh magnetic moment $\left(3.0 \mu_{\mathrm{B}}\right)$ was found by Kim and Takahashi [11] in Fe films containing $\mathrm{Fe}_{16} \mathrm{~N}_{2}$ compound. Therefore, the covalency effects mentioned above should be taken into account in calculating the I-I and I-S interaction energies in the transition metal alloys.

The method we will use in this study closely parallels that developed by Einstein and Schrieffer for the adatom interactions. Since we are interested in covalent binding between interstitial solute atom and the host metal atom (e.g. $\mathrm{H}, \mathrm{C}, \mathrm{N}, \mathrm{O}$ in $\mathrm{Fe}, \mathrm{Co}, \mathrm{Ni}, \mathrm{Nb}$, $\mathrm{Ta}, \mathrm{Pd}, \ldots)$, we will give here a formulation for transition metal (host) systems. For simplicity, we have allowed ourselves a number of simplifying assumptions. The interstitial solute atoms are assumed to have a single energy level, a reasonable approximation for a simple gas atom (or an atom with partially filled d shell; Anderson model [12]). The approximation of a single-site potential in the Wannier representation is used for the substitutional solute atom (Koster-Slater approach). A single band (tightbinding) model is assumed to represent the $d$ bands in transition metals and the sp band is neglected. To study the behaviour of the interstitial solute atom, we consider a perturbed Hamiltonian of the form $\mathscr{H}=H_{0}+V \cdot H_{0}$ is the Hamiltonian for a host metal (d-band) plus the Hamiltonian for the isolated gas atoms, with eigenvalue $\varepsilon_{a}$. The potential $V$ connects the interstitial solute atom $|a\rangle$ with binding sites $|i\rangle, \ldots$, allowing hopping between them. Correlation effects ( $U n_{a \downarrow} n_{a \downarrow} ; U$ is the Coulomb repulsion) are not considered explicitly here, but can be roughly taken into account by self-consistently adjusting the adsorbed atom level. It is well known that this approximation is reasonable in systems where there is only a weak tendency toward localized-moment formation on the adsorbed atoms, that is, $U / \pi . \Gamma<1$, where $\Gamma$ is a measure of the half-width of the density of states at the adsorbed atom (interstitial atom) site.

The format of this paper is as follows. In section 2, we discuss the behaviours of the interstitial atoms and the interaction energy between them in the simple cubic lattice. In section 3, the dependence of lattice structures on the interaction energy is investigated and the direct interaction between interstitial atoms is discussed. The IS interaction is investigated in section 4 . In section 5, the results of the present study are summarized and the possible extension is discussed. In addition, the solute (I-I) interaction calculated on the elastic theory is presented for comparison.

2. General property of interstitial atoms. - We present in this section both the calculational method and results for the simple cubic lattice with nearestneighbour interaction.

2.1. Single INTERSTICIEL ATOM. - In order to perform the numerical calculations, it is necessary to choose a simple method for treating the d electrons in the perfect lattice. The easiest way is to use the tightbinding approximation (TBA) which has proved to be of semi-quantitative value for the calculations of the cohesive energy [13], elastic modulus [14], vacancy formation energy [15], di-vacancy binding energy [16], surface tensions [17], and others. Since these calculations of energies do not depend very much on the details of the density of states curve and of the lattice structure, we will treat a simple cubic lattice with five independent $s$ orbitals per atom to simplify the problem.

Our method to calculate the change in the density of states of a perturbed Hamiltonian $H=H_{0}+V$ closely parallels that developed by Einstein and Schrieffer [8]. The general method can be derived as follows. If we define $G^{0}=\left(\varepsilon-H_{0}-i s\right)^{-1}$, the change in the density of states can be derived as

$$
\begin{aligned}
\Delta \rho(\varepsilon) & =\frac{1}{\pi} \operatorname{Im} \sum_{j}\left(\frac{1}{\varepsilon-\varepsilon_{j}-i s}-\frac{1}{\varepsilon-\varepsilon_{j}^{0}-i s}\right) \\
& =\frac{1}{\pi} \operatorname{Im} \frac{\partial}{\partial \varepsilon} \ln \operatorname{det}\left(\frac{1}{\varepsilon-\mathcal{H}_{0}-i s}\right) \cdot(\varepsilon-\mathcal{H}-i s) \\
& =\frac{1}{\pi} \operatorname{Im} \frac{\partial}{\partial \varepsilon} \ln \operatorname{det}\left(1-G^{0} \cdot V\right)
\end{aligned}
$$

\begin{tabular}{|c|c|c|c|c|c|c|c|c|c|}
\hline Elements & $Y$ & $\mathrm{Sc}$ & $\mathrm{Zr}$ & $\mathrm{Ti}$ & $\mathrm{Hf}$ & $\mathrm{Ta}$ & $\mathrm{Nb}$ & V & $P d$ \\
\hline - & - & - & - & - & - & - & - & - & - \\
\hline
\end{tabular}

TABLE I

Energies of solution of hydrogen atom in transition metals (from ref. [45]) 
where $\varepsilon_{j}^{0}$ and $\varepsilon_{j}$ are the eigenvalues of $\mathcal{H}_{0}$ and $\mathcal{H}$, respectively. The change in the one electron energy, $\Delta W$, is obtained by taking into consideration the change in Fermi energy and the conservation of electrons as

$$
\begin{aligned}
\Delta W & =2 \int_{-\infty}^{\varepsilon_{\mathrm{f}}}\left(\varepsilon-\varepsilon_{\mathrm{f}}\right) \Delta \rho(\varepsilon) \mathrm{d} \varepsilon \\
& =-\frac{2}{\pi} \int_{-\infty}^{\varepsilon_{\mathrm{f}}} \operatorname{Im} \ln \operatorname{det}\left(1-G^{0} \cdot V\right) \mathrm{d} \varepsilon .
\end{aligned}
$$

For the single interstitial atom in the simple cubic lattice, we calculate here only the case where the interstitial atom is located in the bridge-binding sites (see Fig. 1). Although the simple cubic lattice is rare in nature, the interstitial sites (bridge-binding) in this lattice resemble the octahedral sites of the b.c.c. lattice in the sense that they are surrounded by the same number of the nearest host atoms. Therefore it would be useful and informative to investigate the various situations of the interstitial atoms in the simple cubic lattice. In this bridge-binding interstice, $V$ connects the state $|a\rangle$ and states $|1\rangle$ and $|2\rangle$, the Wannier states localized to the nearest-neighbour sites of $a$. Thus det $\left(1-G^{0} V\right)$ is given for the single interstitial atom as

$$
\begin{aligned}
& \operatorname{det}\left(1-G^{0} \cdot V\right)= \\
& =\left|\begin{array}{ccc}
1 & -G_{a a} V_{a 1} & -G_{a a} V_{a 2} \\
-\left(G_{11} V_{1 a}+G_{12} V_{2 a}\right) & 1 & 0 \\
-\left(G_{21} V_{1 a}+G_{22} V_{2 a}\right) & 0 & 1
\end{array}\right| \\
& =1-2 V_{a}^{2}\left(G_{0}+G_{1}\right) G_{a a},
\end{aligned}
$$

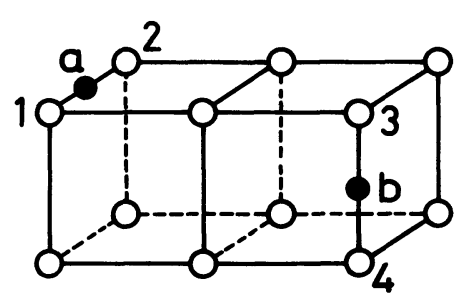

(a)
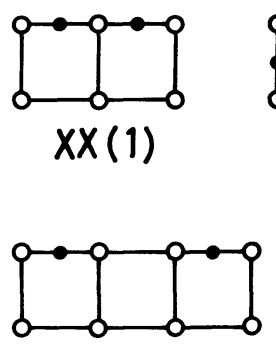

$x \times(2)$

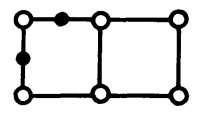

$X Y(1)$

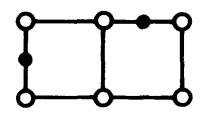

$X Y(2)$

(b)

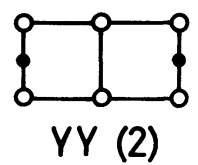

YY (2)

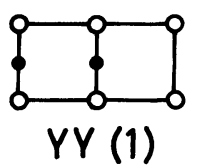

FIG. 1. - Diagrams illustrating the labeling convention for the binding of interstitial pairs $(a)$ in a simple cubic lattice and the six possible types of binding on the $(100)$ plane $(b)$. where

$$
\begin{aligned}
G_{i j} & \equiv G_{i-j}=\frac{1}{N} \sum_{\mathbf{k}} \mathrm{e}^{i \mathbf{k} \cdot \mathbf{R}_{i j}} /\left(\varepsilon-\varepsilon_{\mathbf{k}}-i s\right), \\
G_{a a} & =\left(\varepsilon-\varepsilon_{a}-i s\right)^{-1}
\end{aligned}
$$

Here we have also defined

$$
V_{a}=\left|V_{a 1}\right|=\left|V_{a 2}\right|, \quad V_{1 a}=V_{a 1}^{*}, \quad V_{2 a}=V_{a 2}^{*} .
$$

$\varepsilon_{\mathbf{k}}$ is the eigenvalue of the host metal Hamiltonian. Therefore, we have

$$
\begin{aligned}
& \Delta W_{\mathrm{s}} \equiv \Delta W(\text { single })=-\frac{2}{\pi} \times \\
& \quad \times \int_{-\infty}^{\varepsilon_{\mathrm{f}}} \operatorname{Im} \cdot \ln \left[1-2 V_{a}^{2}\left(G_{0}+G_{1}\right) /\left(\varepsilon-\varepsilon_{a}-i s\right)\right] \mathrm{d} \varepsilon .
\end{aligned}
$$

It must be noted here that expressions (2.3) and (2.4) are rigorous only when $U=0$, since we do not treat Coulomb interaction effects explicitly. Our $\varepsilon_{a}$ is in fact a rather phenomenological parameter which should be rescaled to give at least a Hartree-Fock account of the Coulomb interaction $U$ between an upand a down-spin electron on the interstitial atom. The expression of (2.4) includes the shift in energy due to changes in the density of states of both the interstitial atom and solid when the gas atom is adsorbed. Frequently, it is assumed that the change of density of states in the solid is negligible (the compensation theorem).

Now, we consider the bound (or split off) states, sharply localized energy states lying above or below the continuum band. The position of the bound states, which we denote by $\varepsilon_{\mathrm{bs}}$, is given as

$$
f\left(\varepsilon_{\mathrm{bs}}\right)=\varepsilon_{\mathrm{bs}}-\varepsilon_{a}-2 V_{a}^{2} \operatorname{Re} \cdot\left[G_{0}\left(\varepsilon_{\mathrm{bs}}\right)+G_{1}\left(\varepsilon_{\mathrm{bs}}\right)\right]=0 .
$$

Since $f(\varepsilon)$ is continuous and monotonically decreasing, it must have a single zero below the band when $V_{a}$ exceeds a critical value. Similarly, there can be at most one such bound state above the band. In order to investigate the contribution of a bound state to $\Delta W_{s}$, it is convenient to put $(2.4)$ as

$$
\begin{aligned}
\Delta W_{\mathrm{s}} & =\frac{2}{\pi}\left[\int_{-\infty}^{\varepsilon_{\mathrm{f}}} \arg \left(\varepsilon-\varepsilon_{a}-i s\right) \mathrm{d} \varepsilon-\right. \\
& \left.-\int_{-\infty}^{\varepsilon_{\mathrm{f}}} \arg \left(\varepsilon-\varepsilon_{a}-2 V_{a}^{2}\left(G_{0}+G_{1}\right)-i s\right) \mathrm{d} \varepsilon\right] .
\end{aligned}
$$

If there is a bound state below the band, the integrands do not cancel between $\varepsilon_{\mathrm{bs}}$ and $\varepsilon_{0}$ (bottom of the band; $-\frac{1}{2} W_{\mathrm{b}}$ (bandwidth)), and thus (2.4) can be written

$$
\begin{aligned}
\Delta W_{\mathrm{s}}= & 2\left(\varepsilon_{\mathrm{bs}}-\varepsilon_{0}\right)-\frac{2}{\pi} \times \\
& \times \int_{\varepsilon_{0}}^{\varepsilon_{\mathrm{f}}} \arg \left\{1-\frac{2 V_{a}^{2}\left(G_{0}+G_{1}\right)}{\varepsilon-\varepsilon_{a}-i s}\right\} \mathrm{d} \varepsilon .
\end{aligned}
$$


We see here that as $V_{a}$ grows very large, $\varepsilon_{\mathrm{bs}}$ approaches $-\sqrt{2} V_{a}$ asymptotically, so that $\Delta W_{\mathrm{s}} \approx-2 \sqrt{2} V_{a}$ ( $\varepsilon_{\mathrm{bs}}$ lies in the asymptotic region of the unperturbed Green's function). In this limiting case, $\Delta W_{s}$ becomes independent of the bandwidth of the host metal, so that the host band appears to the interstitial atom as essentially a single energy level.

In order to perform the numerical calculation we further need the values of $\varepsilon_{a}$ and $V_{a}$. Since it is difficult to determine $\varepsilon_{a}$ for specific systems, we often replace it by the occupation number of the interstitial atom $\left\langle n_{a}\right\rangle$ in the following discussions,

$$
\left\langle n_{a}\right\rangle=\frac{1}{\pi} \int_{-\infty}^{\varepsilon_{\mathrm{f}}} \operatorname{Im} .\left(\varepsilon-\varepsilon_{a}-2 V_{a}^{2}\left(G_{0}+G_{1}\right)-i s\right)^{-1} \mathrm{~d} \varepsilon .
$$

The value of $n_{a}$ could be deduced from experimental results on the ionization state of the interstitially dissolved atoms [18], especially when the interstitial atom is $\mathrm{H}$ atom. Since it is difficult to estimate our $V_{a}$ from first principles (particularly when $\mathrm{d}$ orbitals are involved), we develop here a simple method to estimate $V_{a}$. In the problem of adatom interaction (chemisorption), the interaction strength is often deduced $[7,8]$ from the observed chemisorption energy. In the following estimation of $V_{a}$, we assume that the metal has a single $s$ band, which is treated in the TBA (as mentioned before). All atomic orbitals are of simple Gaussian form :

$$
\varphi_{i}(\mathbf{r})=\left(2 \alpha_{1} / \pi\right)^{3 / 4} \exp \left(-\alpha_{1} \cdot\left|\mathbf{r}-\mathbf{R}_{i}\right|^{2}\right)
$$

for the metal atoms, and

$$
\varphi_{\mathrm{a}}(\mathbf{r})=\left(2 \alpha_{0} / \pi\right)^{3 / 4} \exp \left(-\alpha_{0} \cdot\left|\mathbf{r}-\mathbf{R}_{\mathrm{a}}\right|^{2}\right)
$$

for the adsorbed (interstitial) atoms. The Gaussian size parameter for the adsorbed atom is fixed at the value that minimizes the free gas atom energy. For example, for a free hydrogen atom, $\alpha_{0}$ was chosen as $\alpha_{0}=\left(\frac{8}{9} \pi\right) a_{0}^{-2}$ where $a_{0}$ is the Bohr radius. For simplicity, the average effective potential due to the interstitial solute atom is taken to be of screened Coulomb form and spherically symmetrical:

$$
v(r)=Z^{*} e^{2} \cdot \exp (-q r) / r,
$$

where $Z^{*}$ is an effective charge of the solute ion and $q$ is taken as the Thomas-Fermi screening constant. Thus $V_{a i}$ is given as

$$
\begin{aligned}
& V_{a i}=\langle a|v(r)| i\rangle \\
& \quad=\left(4 \alpha_{0} \alpha_{1} / \pi^{2}\right)^{3 / 4} \cdot \frac{\pi Z^{*} e^{2}}{\alpha_{1} R_{a i}} \frac{\exp \left(-\alpha_{1} \cdot R_{a i}^{2}\right)}{\left(\alpha_{0}+\alpha_{1}\right)^{1 / 2}} \times \\
& \times\left[\exp \frac{\left(q-2 \alpha_{1} R_{a i}\right)^{2}}{4\left(\alpha_{0}+\alpha_{1}\right)} \cdot \operatorname{Erfc}\left\{\frac{q-2 \alpha_{1} R_{a i}}{2\left(\alpha_{0}+\alpha_{1}\right)^{1 / 2}}\right\}\right. \\
& \left.-\exp \frac{\left(q+2 \alpha_{1} R_{a i}\right)^{2}}{4\left(\alpha_{0}+\alpha_{1}\right)} \cdot \operatorname{Erfc}\left\{\frac{q+2 \alpha_{1} R_{a i}}{2\left(\alpha_{0}+\alpha_{1}\right)^{1 / 2}}\right\}\right],
\end{aligned}
$$

where Erfc $(x)$ is the Gauss' error function. The results of $V_{a}=\left|V_{a i}\right|$ for $\mathrm{H}$ atom in some transition metals are listed in table II. In the present calculation, the ratio $\alpha_{1} / \alpha_{0}$ is taken to be 0.25 , that is an interstitial atom whose r.m.s. size is one-half that of the host metal atoms and $Z^{*}$ and $q$ are taken to be 1.0 and $1.0 a_{0}^{-1}$, respectively. Therefore, the specific values in table II should be taken as semi-quantitative. We see from table II that our $V_{a}$ is in the same order (but relatively small) as that for the adatoms. This is not surprising since the adsorbed atom-metal atom distance has almost the identical value for both cases.

We now evaluate the integral $\Delta W_{\mathrm{s}}$ in (2.6) for $V_{a} / 2 T=1 \sim 2$ in view of the results of table II. The energy unit of our calculation is $2 T$, i.e., $\frac{1}{6} W_{\mathrm{b}}$, the natural unit of the tight binding model. The results for $V_{a} / 2 T=1, \varepsilon_{a}=-0.5,0.0$ and 0.5 are plotted in figure 2 . We see that there are no positive (repulsive) peaks and no noticeable substructure. This is due to the fact that the present calculation does not take into account the repulsive short range ion-ion interactions. $\Delta W_{\mathrm{s}}$ takes its minimum (negative) value when $\varepsilon_{\mathrm{f}}=\varepsilon_{a}$. This is because in this case the maximum number of electrons have their energies lowered with few elec-

TABLE II

Calculated $V_{a}$ from (2.8) for the interstitial (hydrogen like) solute atom located at the octahedral site of the b.c.c. lattice

\begin{tabular}{lccccc}
\multicolumn{1}{c}{ Elements } & $\mathrm{V}$ & $\alpha-\mathrm{Fe}$ & $\mathrm{Nb}$ & $\mathrm{Mo}$ & $\mathrm{W}$ \\
Crystal structure & - & - & - & - & - \\
Lattice parameter (A) & b.c.c. & b.c.c. & b.c.c. & b.c.c. & b.c.c. \\
$W_{\mathrm{b}}(\mathrm{d}-$ bandwidth $; \mathrm{eV})$ & 3.028 & 2.866 & 3.301 & 3.147 & 3.165 \\
$V_{a}$ (no lattice distortion ; eV) & $6.664\left(^{d}\right)$ & $6.0\left({ }^{c}\right)$ & $9.2\left({ }^{b}\right)$ & $9.2\left(^{a}\right)$ & $10.5\left(^{a}\right)$ \\
$V_{a}\left(\delta a / a_{0}=0.5(*) ; \mathrm{eV}\right)$ & 2.205 & 2.335 & 2.001 & 2.009 & 2.107 \\
\end{tabular}

(*) The change in the atomic distance (host metal) divided by the unistorted atomic distance is 0.5 .

$\left(^{a}\right)$ Mattheiss, L. F., Phys. Rev. 139A (1965) 1893

$\left({ }^{b}\right)$ Mattheiss, L. F., Phys. Rev. B 1 (1970) 373.

(c) Wood, J. H., Phys. Rev. 126 (1962) 517.

(d) Mattheiss, L. F., Phys. Rev. 134A (1964) 192. 
trons having their energies raised. That is, the lower half of the virtual level is occupied and the upper half is unoccupied. These behaviours are also observed in the case of the b.c.c. lattice (see section 3). When a bound state occurs below or above the band, the $\Delta W_{\mathrm{s}}$

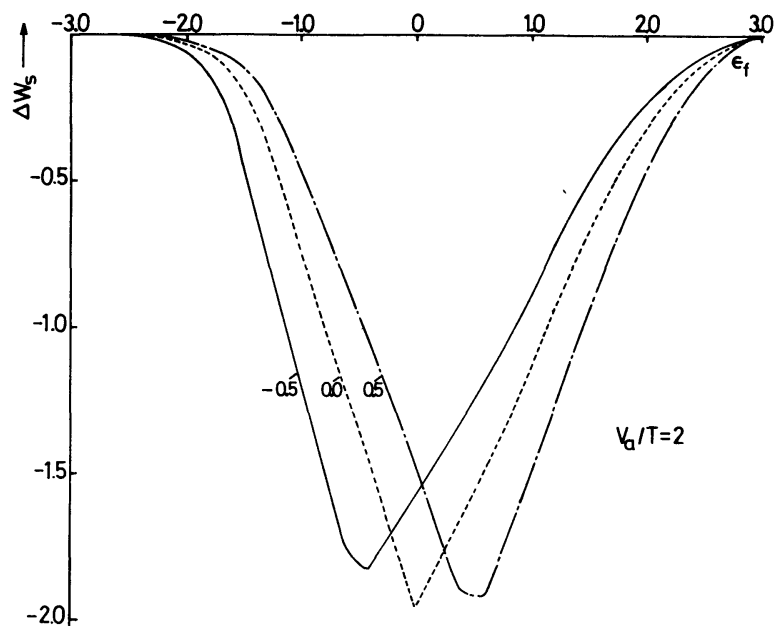

FIG. 2. $-\Delta W_{\mathrm{s}}$ for the bridge-binding site of the simple cubic lattice ; $\varepsilon_{a}=-0.5,0.0$ and 0.5 . The curves indicate the relative insensitivity of $\Delta W_{\mathrm{s}}$ to changes in $\varepsilon_{a}$. curve is negative at the appropriate band edge rather than going to zero there. The critical values of $V_{a}$ for the occurrence of a bound state below (above) the band are 1.92 (1.61), 2.11 (1.49), 2.27 (1.36) for $\varepsilon_{a}=-0.5,0.0$ and 0.5 , respectively.

Finally, it must be noted that our general feature of $\Delta W_{\mathrm{s}}$ is significantly different from that predicted by the screened proton model [19], which predicts that the heat of solution of hydrogen atom is directly dependent on the density of states at the Fermi energy. Since this screened proton model does not take into account the change in the density of states of the host metal and the gas atom upon adsorption, it cannot be used particularly for the range of large $V_{a}$, where the bound states occur and the strong metal-hydrogen bond is formed. The various proton models of hydrogen adsorption were also criticized by Burch [20]. We present the local density of states for the metal atoms neighbouring the interstitial solute atom in figure 10 in the appendix.

2.2 PAIR interstitial SOlUte ATOMS. - In the two-interstitial solute atom (at positions $R_{a}$ and $R_{b}$ ) case we find that

$$
\begin{aligned}
& \operatorname{det}\left(1-G^{0} \cdot V\right)= \\
& \begin{array}{l}
=\left|\begin{array}{cccccc}
1 & -G_{a a} V_{a 1} & -G_{a a} V_{a 2} & 0 & 0 & 0 \\
-\left(G_{11} V_{1 a}+G_{12} V_{2 a}\right) & 1 & 0 & -\left(G_{13} V_{3 b}+G_{14} V_{4 b}\right) & 0 & 0 \\
-\left(G_{21} V_{1 a}+G_{22} V_{2 a}\right) & 0 & 1 & -\left(G_{23} V_{3 b}+G_{24} V_{4 b}\right) & 0 & 0 \\
0 & 0 & 0 & 1 & -G_{b b} V_{b 3} & -G_{b b} V_{b 4} \\
-\left(G_{31} V_{1 a}+G_{32} V_{2 a}\right) & 0 & 0 & -\left(G_{33} V_{3 b}+G_{34} V_{4 b}\right) & 1 & 0 \\
-\left(G_{41} V_{1 a}+G_{42} V_{2 a}\right) & 0 & 0 & -\left(G_{43} V_{3 b}+G_{44} V_{4 b}\right) & 0 & 1
\end{array}\right| \\
=\left\{1-2 r_{a}^{2}\left(G_{0}+G_{1}\right) G_{a a}\right\} \cdot\left\{1-2 V_{b}^{2}\left(G_{0}+G_{1}\right) G_{b b}\right\}-V_{a}^{2} V_{b}^{2} G_{a a} G_{b b}\left(G_{13}+G_{14}+G_{23}+G_{24}\right)^{2} .
\end{array}
\end{aligned}
$$

In this formulation, we see that an adsorbed atom electron is able to hop to the nearest metal atom (1 or 2), propagate to the metal atom nearest the adsorbed atom $b$, hop out to this second energy level, interact, and hop onto the original adsorbed atom a through the reversed hopping path. The pair interaction energy between two interstitial atoms is obtained by symmetry as follows :

$$
\begin{aligned}
\Delta W_{\mathrm{p}}=\Delta W(\text { pair }) & =-\frac{2}{\pi} \int_{-\infty}^{\varepsilon_{\mathrm{f}}} \operatorname{Im} \ln \left[1-\frac{V_{a}^{4}\left(G_{13}+G_{14}+G_{23}+G_{24}\right)^{2}}{\left\{\varepsilon-\varepsilon_{a}-2 V_{a}^{2}\left(G_{0}+G_{1}\right)\right\}^{2}}\right] \mathrm{d} \varepsilon \\
& \cong \frac{2}{\pi} \int_{-\infty}^{\varepsilon_{\mathrm{f}}} \operatorname{Im} \frac{V_{a}^{4}\left(G_{13}+G_{14}+G_{23}+G_{24}\right)^{2}}{\left\{\varepsilon-\varepsilon_{a}-2 V_{a}^{2}\left(G_{0}+G_{1}\right)\right\}^{2}} \mathrm{~d} \varepsilon .
\end{aligned}
$$

The expression of $(2.10 b)$ is a reasonable approximation when

$$
\left|V_{a}^{4}\left(G_{13}+G_{14}+G_{23}+G_{24}\right)^{2} G_{a a}^{2}\right| \ll 1
$$

and is analogous to the first-order expression derived by Kim and Nagaoka [21] for two magnetic impurity atoms. Here it must be noted that the $\varepsilon_{a}$ in $(2.10 a)$ and $(2.10 b)$ is in fact a rather phenomenological parameter which should be rescaled to give at least a Hartree-Fock account of the Coulomb repulsion (as has been already mentioned in the previous section). In this approximation, $\varepsilon_{a}$ is clearly increased by $U$ times the occupation number of the interstitial atom (not equal to that of the isolated interstitial atom) for either spin direction; to lowest order this occupation number can be replaced by that for the isolated interstitial atom [22]. This fact (cancellation) is fortunate if one is trying to introduce the parameter $\left\langle n_{a}\right\rangle$, occupation number of the interstitial 
atom (non-magnetic), instead of the parameter $\varepsilon_{a}$ since $\varepsilon_{a}$ is the most difficult to our parameters to determine. This corresponds to the fact that we do not need to take into account the change in impurity potentials due to the interaction in calculating the electronic pair energy within a tight-binding (Koster-
Slater) model [46]. Thus the pair interaction energy can be obtained by knowing (or as a function of) $\left\langle n_{a}\right\rangle$ without the explicit knowledge of $U$.

Now consider the contribution of the bound states to the pair interaction energy. As in the case of the single solute atom, we decompose $(2.10 a)$ as

$$
\begin{aligned}
& \Delta W_{\mathrm{p}}=-(2 / \pi) \int_{-\infty}^{\varepsilon_{\mathrm{f}}}\left[\arg \left\{\varepsilon-\varepsilon_{a}-2 V_{a}^{2}\left(G_{0}+G_{1}+G_{\mathrm{s}} / 2\right)-i s\right\}+\right. \\
& \left.\quad+\arg \left\{\varepsilon-\varepsilon_{a}-2 V_{a}^{2}\left(G_{0}+G_{1}-G_{s} / 2\right)-i s\right\}-2 \arg \left\{\varepsilon-\varepsilon_{a}-2 V_{a}^{2}\left(G_{0}+G_{1}\right)-i s\right\}\right] \mathrm{d} \varepsilon,
\end{aligned}
$$

where $G_{s}=G_{13}+G_{14}+G_{23}+G_{24}$. It would be seen here that all three arguments will be negative below the band and contribute an integrand of $-\pi$ leading to cancellation. For sufficiently strong $V_{a}$, however, the real parts (of $\{\cdots\}$ ) will become positive below the lower band edge and such a cancellation will not occur. In this case, the contribution of bound states to the pair interaction energy is

$$
-2\left[\left(\varepsilon_{0}-\varepsilon_{\mathrm{bs}}^{+}\right)+\left(\varepsilon_{0}-\varepsilon_{\mathrm{bs}}^{-}\right)-2\left(\varepsilon_{0}-\varepsilon_{\mathrm{bs}}\right)\right]
$$

when $\varepsilon_{\mathrm{f}}$ is within the band. Here $\varepsilon_{\mathrm{bs}}^{+(-)}$is defined by

$$
\varepsilon_{\mathrm{bs}}^{+(-)}-\varepsilon_{a}-2 V_{a}^{2} \operatorname{Re}\left(G_{0}+G_{1}+(-) G_{s} / 2\right)_{\varepsilon_{\mathrm{bs}}^{+(-)}}=0,
$$

$\varepsilon_{\mathrm{bs}}^{+(-)}<\varepsilon_{0}$. Thus $(2.10 a)$ can be rewritten as

$$
\Delta W_{\mathrm{p}}=2\left(\varepsilon_{\mathrm{bs}}^{+}+\varepsilon_{\mathrm{bs}}^{-}-2 \varepsilon_{\mathrm{bs}}\right)-\frac{2}{\pi} \int_{\varepsilon_{0}}^{\varepsilon_{\mathrm{r}}} \arg \left\{1-V_{a}^{4} G_{s}^{2} /\left[\varepsilon-\varepsilon_{a}-2 V_{a}^{2}\left(G_{0}+G_{1}\right)\right]^{2}\right\} \mathrm{d} \varepsilon .
$$

Here $\varepsilon_{\mathrm{bs}}^{+(-)}$or $\varepsilon_{\mathrm{bs}}$ is taken to be $\varepsilon_{0}$ if there is no zero below the band in any one of the three real parts in the arguments in (2.11). The threshold values of $V_{a}$ for occurrence of the bound states denoted by $\varepsilon_{\mathrm{bs}}^{+}$, $\varepsilon_{\mathrm{bs}}$ and $\varepsilon_{\mathrm{bs}}^{-}$are for example 2.95, 1.61 and 1.23, respectively for the nearest-neighbour pair interaction with $\varepsilon_{a}=-0.5$. From the expression of (2.12), we easily obtain the $\Delta W_{\mathrm{p}}$ for very large $V_{a}$. As $V_{a}$ becomes very large, the integral term of $(2.12)$ becomes independent of $V_{a}$, with the value $I_{\mathrm{p}}$

$I_{\mathrm{p}}=-\frac{2}{\pi} \int_{\varepsilon_{0}}^{\varepsilon_{\mathrm{f}}} \arg \left\{1-G_{s}^{2} / 4\left(G_{0}+G_{1}\right)^{2}\right\} \mathrm{d} \varepsilon$.

To analyze the bound state term, it is convenient to write $\operatorname{Re} G_{i j}$ in terms of a moment expansion :

$$
\operatorname{Re} . G_{i j} \sim \frac{1}{\varepsilon} \cdot \sum_{n=0}^{\infty} \mu_{i j}(n) / \varepsilon^{n} \text { for }|\varepsilon|>\left|\varepsilon_{0}\right|
$$

where

$$
\mu_{i j}(n)=\frac{1}{\pi} \int_{\varepsilon_{0}}^{\text {top }}\left(\varepsilon^{\prime}\right)^{n} \operatorname{Im} G_{i j}\left(\varepsilon^{\prime}\right) \mathrm{d} \varepsilon^{\prime}
$$

is called the $n$-th moment of $\operatorname{Im} G_{i j}$. For a periodic lattice with one atom per unit cell, we obtain at once the first moments of $\mu_{i i}(n): \mu_{i i}(0)=1, \mu_{i i}(1)=\alpha+E_{0}$, $\mu_{i i}(2)=Z T^{2}$, where $Z$ is the coordination number of the lattice and $\alpha+E_{0}$ is the energy shift of the tightbinding band. Let $\mu_{s}(n)$ be defined as

$$
\mu_{s}(n)=\mu_{13}(n)+\mu_{14}(n)+\mu_{23}(n)+\mu_{24}(n) .
$$

Then we easily find that

$$
\begin{aligned}
2\left(\varepsilon_{\mathrm{bs}}^{+}+\varepsilon_{\mathrm{bs}}^{-}\right. & \left.-2 \varepsilon_{\mathrm{bs}}\right) \approx-2 V_{a} \times \\
& \times\left[\left\{2+\mu_{s}(0)\right\}^{1 / 2}+\left\{2-\mu_{s}(0)\right\}^{1 / 2}-2 \sqrt{2}\right]
\end{aligned}
$$

for large $V_{a}$. We thus see that when $\mu_{s}(0) \neq 0$ and $V_{a} \gg W_{\mathrm{b}}, \Delta W_{\mathrm{p}}$ asymptotically approaches the above value and when $\mu_{s}(0)=0$ it approaches

$$
I_{\mathrm{p}}\left(I_{\mathrm{p}}+0\left(\mu_{s}^{2}(1) / V_{a}\right)\right) .
$$

The other important feature of the pair interaction is that $\Delta W_{\mathrm{p}}$ vanishes when $\varepsilon_{\mathrm{f}} \rightarrow+\infty$. Essentially, this arises from the fact that the perturbing potential is purely off-diagonal in the site representation. In this case $\operatorname{Tr} \mathscr{H}=\operatorname{Tr} \mathscr{H}_{0}$, therefore there is no interaction energy if all band levels are occupied, even though individual levels may shift. Although this result is of such importance and utility, we will not present the explicit proof here since the similar proof has been already given in the problem of chemisorption [8]. This general result is particularly helpful when performing actual numerical calculations.

Now we compute $\Delta W_{\mathrm{p}}$ for certain types of the pair interaction energies. Again we evaluate the integral 
TABLE III

Analytic expressions for the Green's functions $\left(G_{s}^{+}\right.$and $\left.G_{s}^{-}\right)$

$$
\begin{aligned}
& G_{s}^{+}-\frac{1}{3}(2+\varepsilon)+\frac{1}{3}\left(4+2 \varepsilon+\varepsilon^{2}\right) G_{0}-\frac{2}{3} \varepsilon\left(5-\varepsilon^{2}\right) G_{0}^{\prime}+\frac{1}{3}\left(9-10 \varepsilon^{2}+\varepsilon^{4}\right) G_{0}^{\prime \prime} \\
& G_{s}^{-} \quad \frac{\varepsilon}{3}+\frac{1}{3}\left(2-\varepsilon^{2}\right) G_{0}+\frac{2}{3} \varepsilon\left(5-\varepsilon^{2}\right) G_{0}^{\prime}-\frac{1}{3}\left(9-10 \varepsilon^{2}+\varepsilon^{4}\right) G_{0}^{\prime \prime} \\
& \mathrm{XX} \\
& G_{s}^{+}-\frac{1}{3}\left(3+2 \varepsilon+2 \varepsilon^{2}\right)+\frac{1}{6}\left(4+2 \varepsilon+4 \varepsilon^{2}+5 \varepsilon^{3}\right) G_{0}+\frac{1}{6}\left(9-40 \varepsilon-80 \varepsilon^{2}+8 \varepsilon^{3}+15 \varepsilon^{4}\right) G_{0}^{\prime}+\frac{11}{6}\left(9-10 \varepsilon^{2}+\varepsilon^{4}\right) G_{0}^{\prime \prime} \\
& G_{s}^{-} \quad \frac{1}{3}\left(1+2 \varepsilon+2 \varepsilon^{2}\right)+\frac{1}{6}\left(8+2 \varepsilon-4 \varepsilon^{2}-5 \varepsilon^{3}\right) G_{0}-\frac{1}{6}\left(9-40 \varepsilon-80 \varepsilon^{2}+8 \varepsilon^{3}+15 \varepsilon^{4}\right) G_{0}^{\prime}-\frac{11}{6}\left(9-10 \varepsilon^{2}+\varepsilon^{4}\right) G_{0}^{\prime \prime} \\
& G_{s}^{+}-\frac{2}{3}+\frac{1}{12}(17+8 \varepsilon) G_{0}+\frac{1}{6} \varepsilon\left(5-\varepsilon^{2}\right) G_{0}^{\prime}-\frac{1}{12}\left(9-10 \varepsilon^{2}+\dot{\varepsilon}^{4}\right) G_{0}^{\prime \prime} \\
& G_{s}^{-} \quad \frac{7}{12} G_{0}-\frac{1}{6} \varepsilon\left(5-\varepsilon^{2}\right) G_{0}^{\prime}+\frac{1}{12}\left(9-10 \varepsilon^{2}+\varepsilon^{4}\right) G_{0}^{\prime \prime} \\
& 2 \\
& G_{s}^{+}-\frac{1}{3}(1+\varepsilon)+\frac{1}{24}\left(18+10 \varepsilon+8 \varepsilon^{2}-\varepsilon^{3}\right) G_{0}-\frac{1}{24}\left(9+60 \varepsilon-40 \varepsilon^{2}-12 \varepsilon^{3}+7 \varepsilon^{4}\right) G_{0}^{\prime}-\frac{1}{24}(1-4 \varepsilon)\left(9-10 \varepsilon^{2}+\varepsilon^{4}\right) G_{0}^{\prime \prime} \\
& G_{s}^{-}-\frac{1}{3}(1-\varepsilon)+\frac{1}{24}\left(30+6 \varepsilon-8 \varepsilon^{2}+\varepsilon^{3}\right) G_{0}+\frac{1}{24}\left(9+60 \varepsilon-40 \varepsilon^{2}-12 \varepsilon^{3}+7 \varepsilon^{4}\right) G_{0}^{\prime}+\frac{1}{24}(1-4 \varepsilon)\left(9-10 \varepsilon^{2}+\varepsilon^{4}\right) G_{0}^{\prime \prime} \\
& G_{s}^{+}-\frac{2}{3}+\frac{1}{6}(5+4 \varepsilon) G_{0}+\frac{1}{3} \varepsilon\left(5-\varepsilon^{2}\right) G_{0}^{\prime}-\frac{1}{6}\left(9-10 \varepsilon^{2}+\varepsilon^{4}\right) G_{0}^{\prime \prime} \\
& G_{s}^{-} \quad \frac{7}{6} G_{0}-\frac{1}{3} \varepsilon\left(5-\varepsilon^{2}\right) G_{0}^{\prime}+\frac{1}{6}\left(9-10 \varepsilon^{2}+\varepsilon^{4}\right) G_{0}^{\prime \prime} \\
& G_{s}^{+}-\frac{2}{3} \varepsilon+\frac{1}{12}\left(8+2 \varepsilon+8 \varepsilon^{2}-\varepsilon^{3}\right) G_{0}-\frac{1}{12}\left(9+80 \varepsilon-40 \varepsilon^{2}-16 \varepsilon^{3}+7 \varepsilon^{4}\right) G_{0}^{\prime}+\frac{1}{12}(4 \varepsilon+1)\left(9-10 \varepsilon^{2}+\varepsilon^{4}\right) G_{0}^{\prime \prime} \\
& G_{s}^{-}-\frac{2}{3}(1-\varepsilon)+\frac{1}{12}\left(16+6 \varepsilon-8 \varepsilon^{2}+\varepsilon^{3}\right) G_{0}+\frac{1}{12}\left(9+80 \varepsilon-40 \varepsilon^{2}-16 \varepsilon^{3}+7 \varepsilon^{4}\right) G_{0}^{\prime}-\frac{1}{12}(4 \varepsilon+1)\left(9-10 \varepsilon^{2}+\varepsilon^{4}\right) G_{0}^{\prime \prime}
\end{aligned}
$$

$G_{0}^{\prime}$ and $G_{0}^{\prime \prime}$ are also expressed in terms of the complete elliptic integrals of the first and the second kind (ref. [24]).

$\Delta W_{\mathrm{p}}$ in (2.11) for $V_{a} / 2 T=1 \sim 2$. For simplicity and convenience (comparison with the adatom interaction in ref. [8]), we calculate here the pair interaction energies only in the case of the interstitials located on the (100) plane of a simple cubic lattice. Furthermore, we only consider three types of the interactions, $\mathrm{XX}, \mathrm{XY}$ and $\mathrm{YY}$ interaction as defined in figure 1 . In order to perform the numerical calculations, we need the values of the unperturbed (lattice) Green's function for any $\varepsilon$ in (2.11). Recently, Morita [23] showed that the lattice Green's function $G_{l m n}$ for an arbitrary site (lmn) for the simple cubic lattice is able to be expressed in terms only of $G_{0}, G_{2}$ and $G_{3}$. Horiguchi and Morita [24] have further shown that $G_{2}$ and $G_{3}$ are expressed in the form of a sum of products of the complete elliptic integrals of the first and the second kind $(K(k)$ and $E(k))$. Therefore $G_{l m n}$ for the arbitrary site (lmn) can be expressed in the above form. Since a powerful method for calculating $K(k)$ and $E(k)$ in the complex $k$ plane has been developed [27], we express the Green's functions

$$
G_{s}^{+(-)}=G_{0}+G_{1}+(-) G_{s} / 2
$$

in table III for three types of pair interactions in terms of $G_{0}, G_{0}^{\prime}$ and $G_{0}^{\prime \prime}$, where $G_{0}^{\prime}$ and $G_{0}^{\prime \prime}$ are the first and second derivative of $G_{0}$ with respect to $\varepsilon$, respectively. The results of the numerical calculations of $\Delta W_{\mathrm{p}}$ for $\mathrm{XY}(1), \mathrm{XX}(1)$ and $\mathrm{YY}(1)$ interaction $\left(V_{a} / T=2\right)$ are plotted in figures $3 a, 3 b$ and $3 c$, respectively. For small shifts in $\varepsilon_{a}$, and for both $\varepsilon_{\mathrm{f}}$ and $\varepsilon_{a}$ not so close to a band edge, we can say that $\Delta W_{\mathrm{p}}$ is locally well approximated by a function of only $\varepsilon_{\mathrm{f}}-\varepsilon_{a}$. The weak dependence of $\Delta W_{\mathrm{p}}$ on $\varepsilon_{a}$ (compared with $\left.\varepsilon_{\mathrm{f}}\right)\left({ }^{1}\right)$ is fortunate if one is trying to find the appropriate calculated curve for experimentally determined values of input parameters $\varepsilon_{a}, \varepsilon_{\mathrm{f}}, W_{\mathrm{b}}$ and $V_{a}$ since $\varepsilon_{a}$ is the most difficult to determine. For stronger potentials, the features of the interaction curve in the interior of the band are relatively insensitive to variations of $V_{a}$. If one characterizes the interaction by the largest absolute magnitude of $\Delta W_{\mathrm{p}}$ for the Fermi energy at any place within the band, one can say something meaningful about the decrease in the interaction energy with distance (for potentials sufficiently weak so that no bound states occur). Our calculations of $\Delta W_{\mathrm{p}}$ along the $\langle 100\rangle$ direction suggest that $\Delta W_{\mathrm{p}} \propto R^{-3}$ even for the neighbouring pairs (separated by $1 \sim 3$ lattice constants). For the nearest-neighbour pair interaction $(X Y(1))$, we see that $\Delta W_{\mathrm{p}}$ varies in sequences of negative-positivenegative (in a rather predictable fashion) and

${ }^{1}{ }^{1}$ In the unphysical limit of very strong potential $V_{a}$, the dependence of $\Delta W_{\mathrm{p}}$ on $\varepsilon_{a}$ vanishes entirely. 
$\left|\Delta W_{\mathrm{p}}\right| \lesssim 0.2$ for $V_{a} / T=2$ and $\varepsilon_{a}$ not so close to a band edge. We then find that the maximum value of $\mid \Delta W_{\mathrm{p}}$ (N.N.) $\mid$ is about $0.3 \mathrm{eV}$ for $W_{\mathrm{b}}=9 \mathrm{eV}$ and $V_{a}=1.5 \mathrm{eV}$. This indicates the importance of the

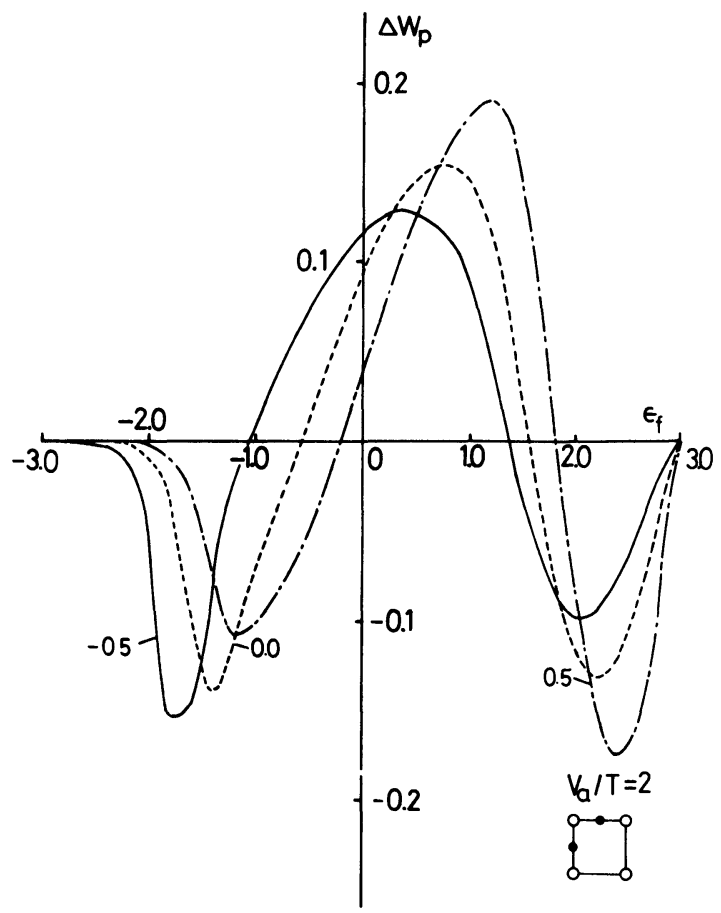

FIG. 3a. - XY(1) pair-interaction energy for $V_{a} / T=2, \varepsilon_{a}=-0.5$, 0.0 and 0.5 .

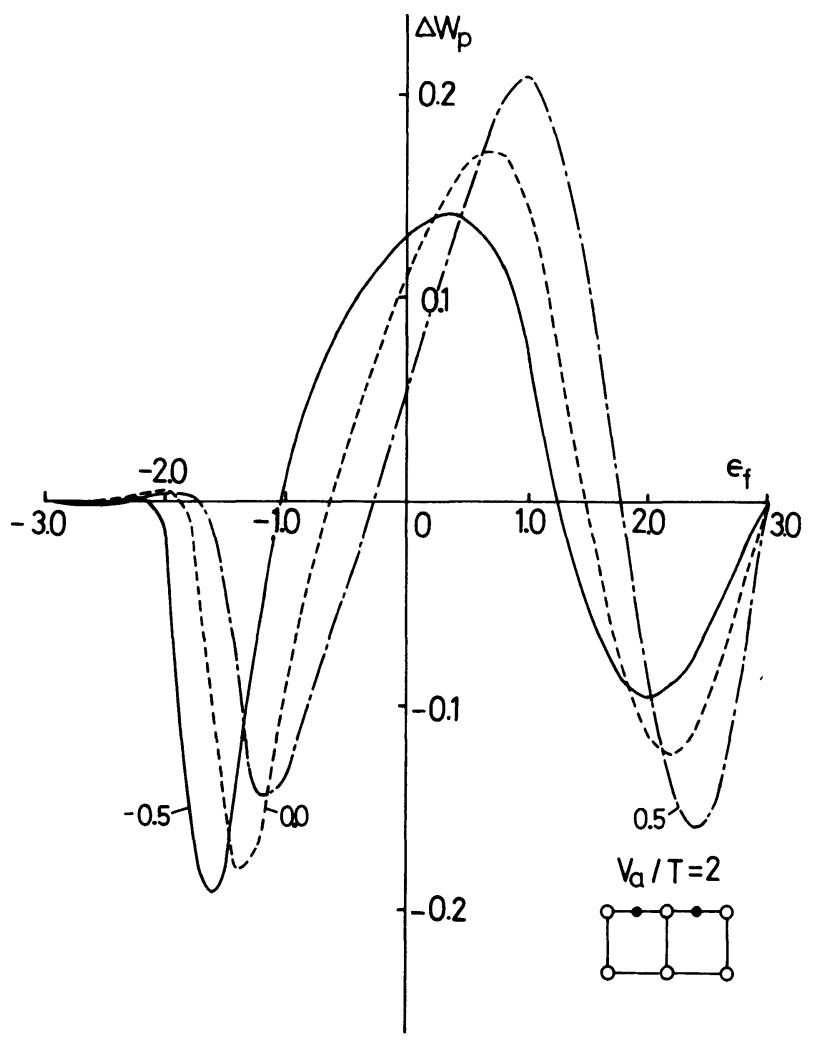

FIG. $3 b .-\mathrm{XX}(1)$ pair-interaction energy for $V_{a} / T=2, \varepsilon_{a}=-0.5$, 0.0 and 0.5 .

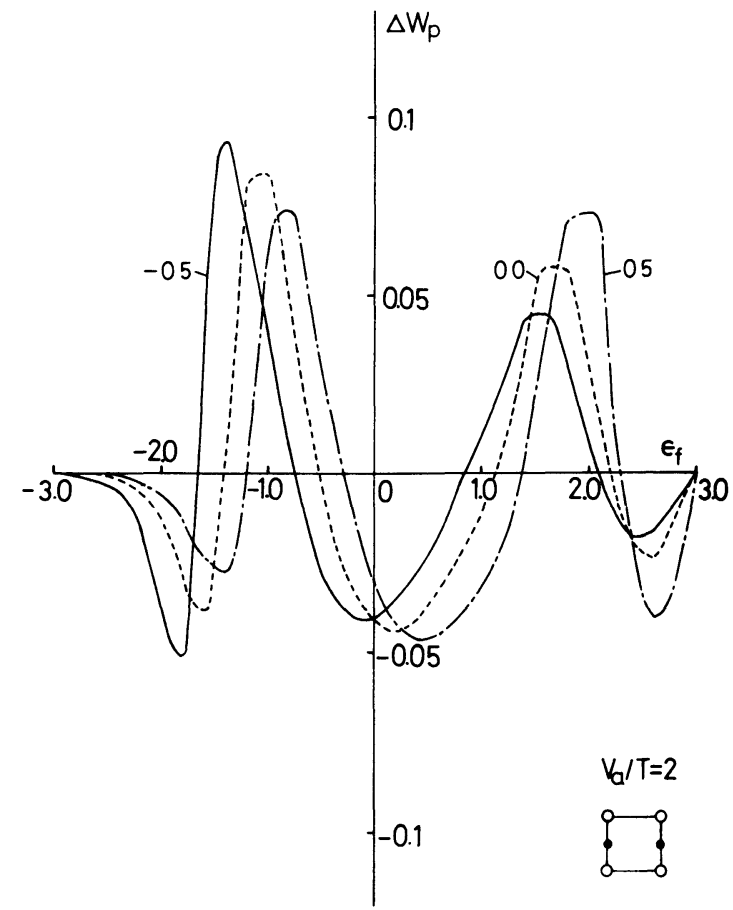

FIG. 3c. - YY(1) pair-interaction energy for $V_{a} / T=2, \varepsilon_{a}=-0.5$, 0.0 and 0.5

electronic contribution to the pair interaction energies $\left(\begin{array}{l}2 \\ 2\end{array}\right)$. Finally, it must be noted that the direct interaction between interstitial atoms can not be in general neglected for the nearest-neighbour pair. The brief discussion on this matter will be given in section 3 .

3. Variation of the crystal structure. - In order to represent interstitials like hydrogen atom in the b.c.c. metals, which are known to be located at tetrahedral interstices [31], more properly we further study the behaviours of the interstitials in this lattice using the tight-binding $\mathrm{s}$ band with nearest-neighbour interaction. For the nearest-neighbour pair interaction, we take into account the direct interaction (hopping) term between the interstitial solute atoms.

3.1 Single INTERSTItial ATOM IN b.c.c. Metals. There can be two kinds of the interstices in the b.c.c. lattice, octahedral and tetrahedral sites with 2 and 4 nearest-neighbour host atoms, respectively. The first and the second nearest-neighbour distance between the interstitial atom located at the octahedral site and the host metal atom are $0.5 a$ and $a / \sqrt{2}(=0.71 a)$, respectively, where $a$ is the lattice constant. For the tetrahedral sites, the first and the second nearestneighbour distance are $\sqrt{5 / 4}(=0.56) a$ and $\sqrt{13} / 4(=0.9) a$, respectively. Therefore, the assumption of taking into account only the nearest-neighbour

$\left(^{2}\right)$ In general, the magnitude of the interaction energy between interstitial $(\mathrm{H}, \mathrm{C}, \mathrm{N}, \mathrm{O})$ atoms in transition metals is roughly $0.3 \mathrm{eV}$ at most. 
hopping potential would certainly be justified. The expression of det $\left(1-G^{0} . V\right)$ for the single solute atom in the octahedral interstices is obtained by just replacing $G_{100}$ by $G_{200}$ (this replacement is of course the conventional one) in (2.3). For the tetrahedral interstices, the unperturbed Green's functions can be written in the matrix form as

$$
G^{0}=\left|\begin{array}{ccccc}
G_{a a} & 0 & 0 & 0 & 0 \\
0 & G_{0} & G_{2} & G_{111} & G_{111} \\
0 & G_{2} & G_{0} & G_{111} & G_{111} \\
0 & G_{111} & G_{111} & G_{0} & G_{2} \\
0 & G_{111} & G_{111} & G_{2} & G_{0}
\end{array}\right|
$$

The perturbing potential is of the following matrix form

$$
V=\left|\begin{array}{ccccc}
0\left(\text { or } \delta V_{\mathrm{A}}\right) & V_{a 1} & V_{a 1^{\prime}} & V_{a 2} & V_{a 2^{\prime}} \\
V_{1 a} & 0 & 0 & 0 & 0 \\
V_{1^{\prime} a} & 0 & 0 & 0 & 0 \\
V_{2 a} & 0 & 0 & 0 & 0 \\
V_{2^{\prime} a} & 0 & 0 & 0 & 0
\end{array}\right|,
$$

where the $V_{a a}$ component has the non-vanishing value $\delta V_{\mathrm{A}}$ when $U \neq 0$. Because of the symmetry of $V$, it may be block diagonalized by the basis states transformation as discussed by Allan [25] and Wolfram and Callaway [26]. Under this transformation, $G^{0}$ and $V$ have the following forms:

$$
\begin{gathered}
G^{0}=\left|\begin{array}{ccccc}
G_{a a} & 0 & 0 & 0 & 0 \\
0 & G_{0}+G_{2}+2 G_{111} & 0 & 0 & 0 \\
0 & 0 & G_{0}+G_{2}-2 G_{111} & 0 & 0 \\
0 & 0 & 0 & & G_{0}-G_{2} \\
0 & 0 & 0 & 0 & G_{0}-G_{2}
\end{array}\right|, \\
V=\left|\begin{array}{ccccc}
0\left(\text { or } \delta V_{\mathrm{A}}\right) & -2 V_{a} & 0 & 0 & 0 \\
-2 V_{a} & 0 & 0 & 0 & 0 \\
0 & 0 & 0 & 0 & 0 \\
0 & 0 & 0 & 0 & 0 \\
0 & 0 & 0 & 0 & 0
\end{array}\right|,
\end{gathered}
$$

where we have used the relation $V_{a 1}=V_{1 a}^{*}, V_{a}=\left|V_{a 1}\right|, V_{a 2}=V_{2 a}^{*}, V_{a}=\left|V_{a 2}\right|, \ldots$ Thus, we easily obtain $\operatorname{det}\left(1-G^{0} . V\right)$ as

$$
\operatorname{det}\left(1-G^{0} \cdot V\right)=1-4 V_{a}^{2} \cdot\left(G_{0}+G_{2}+2 G_{111}\right) G_{a a},
$$

where $G_{i}$ is the shorthand for $G_{i 00}$. For the b.c.c. lattice (s band) with the nearest-neighbour interaction, the lattice Green's function $G_{l m n}$ is defined as

$$
G_{l m n}(\varepsilon)=G_{l m n}=\frac{1}{(2 \pi)^{3}} \int_{-\pi}^{\pi} \mathrm{d} x \int_{-\pi}^{\pi} \mathrm{d} y \int_{-\pi}^{\pi} \mathrm{d} z \frac{\cos (l x) \cos (m y) \cos (n z)}{\varepsilon-i s-\cos x \cos y \cos z},
$$

where $l, m$ and $n$ are all even or all odd integers and the energy unit is the half-bandwidth of the pure metal. The Green's functions $G_{0}, G_{2}$ and $G_{111}$ are expressed in terms of the complete elliptic integral of the first and the second kind, $K(k)$ and $E(k)$ :

$$
\begin{aligned}
G_{0} & =\frac{1}{\varepsilon} \frac{4}{\pi^{2}}[K(k)]^{2}, \\
G_{2} & =\frac{16}{\pi^{2}} \varepsilon\left[E(k)-\left(1-k^{2}\right) \cdot K(k)\right]^{2}, \\
G_{111} & =\varepsilon \cdot G_{0}-1,
\end{aligned}
$$

where $k^{2}=\frac{1}{2}-\frac{1}{2}\left(1-\frac{1}{\varepsilon^{2}}\right) . K(k)$ and $E(k)$ for complex values of $k$ are evaluated with the aid of an efficient procedure of the arithmetic-geometric mean
[27]. The numerical results of $\Delta W_{\mathrm{s}}$ for tetrahedral and octahedral sites with $V_{a}=0.2, \varepsilon_{a}=-0.3$ and 0.3 (energy unit is the half-bandwidth) are shown in figure 4. From figure 4 we see that our calculation of $\Delta W_{\mathrm{s}}$ revealed the general structure of an inverted triangle, smoothed at the base angles (particularly at the lower edge) for both cases of the interstices. As in the case of the interstices of the simple cubic lattice, $\Delta W_{\mathrm{s}}$ takes its largest (negative) value when $\varepsilon_{\mathrm{f}}=\varepsilon_{a}$. This is also due to the fact that the lower half of the virtual level is occupied and the upper half is empty. If one assumes (for comparison) that $V_{a}$ has the same strength for both types of interstices, $\left|\Delta W_{\mathrm{s}}(4)\right|>\left|\Delta W_{\mathrm{s}}(8)\right|$ except for the region $\varepsilon \sim \varepsilon_{0}$ (lower band edge). This assumption would certamly be justified in the case of spherical orbitals and similar interstitial-metal atom distances for the two types of 
binding. Therefore, figure 4 would probably characterize the hydrogen atom in b.c.c. metals better than nitrogen or oxygen atoms (with $\mathrm{p}$ orbitals). The $\mathrm{p}$ orbitals of $\mathrm{C}, \mathrm{N}$ or $\mathrm{O}$ would interact with the directed d-orbitals of host metal atoms. For example, in the case of oxygen adsorption in the $\mathrm{O}_{z}$ octahedral sites (see Fig. 5) of the b.c.c. lattice, a bonding (molecule) orbital would be formed from a combination of the oxygen $P_{z}$ and host metal $d_{3 z^{2}-r^{2}}$ orbitals and the $P_{x}$ and $\mathbf{P}_{y}$ orbitals do not contribute to the bond, to first order. Alternatively, if the oxygen atom is adsorbed in the tetrahedral sites, it seems that the formation of the simple directional bond is impossible and it is expected that the metal-interstitial atom bond would

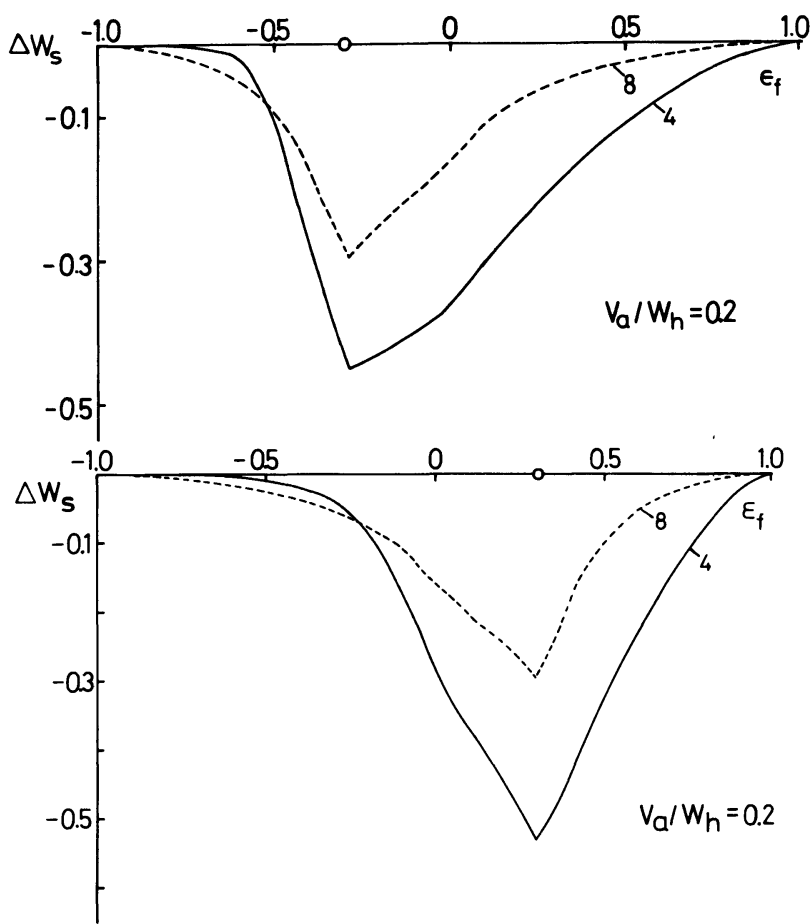

FIG. 4. $-\Delta W_{\mathrm{s}}$ for the tetrahedral interstices (4) and octahedral interstices (8) of the b.c.c. lattice; $\varepsilon_{a}=-0.3$ and 0.3 . $W$ denotes the half-bandwidth of the host metal band.

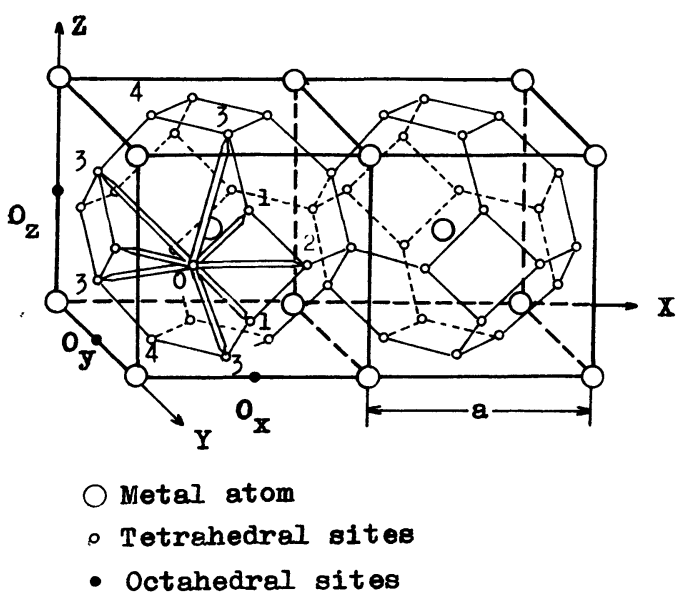

Fig. 5. - Tetrahedral and octahedral interstitial sites in a b.c.c. lattice. Four shells of neighbours (tetrahedral sites) of the site 0 are labelled by 1 to 4 . contain a hybridized orbital of oxygen atom, probably like $\mathrm{sp}_{3}$ hybridized orbital. The numerical results of $\Delta W_{\mathrm{s}}$ in figure 4 clearly demonstrate the difference in the electronic state associated with each site and let one understand the preferential occupancy in the tetrahedral site of the interstitial atoms with spherical atomic orbitals (i.e. $\mathrm{H}$ in $\mathrm{Ta}$ or $\mathrm{Nb}$ ) in the b.c.c. (d-band) metals. This tendency agrees, in appearance, with that obtained by the empirical potential method [28]. However, the definite values of these (electronic) site energies could still not be determined since the one-electron approximation is not a satisfactory basis for calculating the adsorption energy.

The density of states per spin at the interstitial atom site, $\rho_{a}(\varepsilon)$, is given as

$$
\rho_{a}(\varepsilon)=\frac{1}{\pi} \operatorname{Im} g_{a a}(\varepsilon)
$$

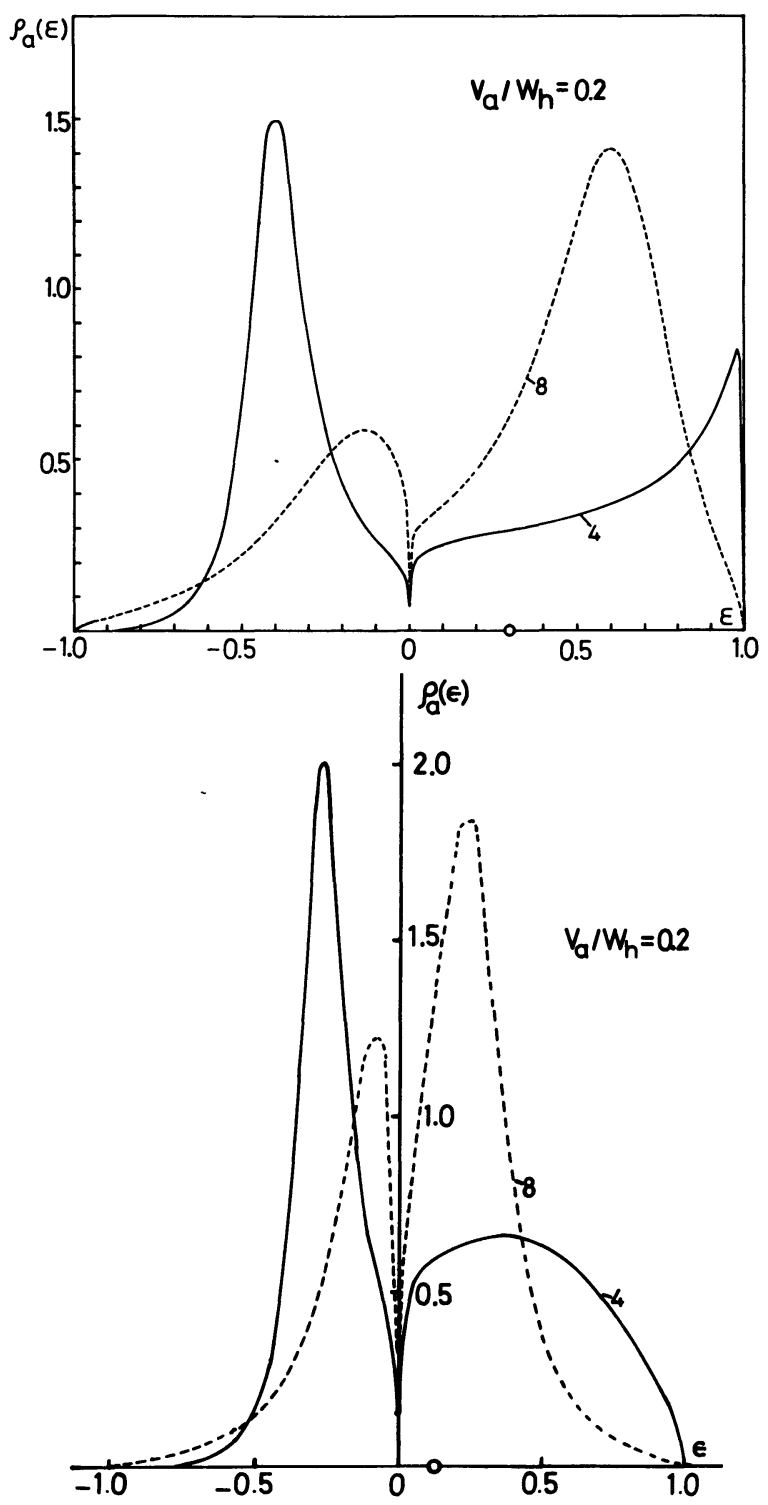

Fig. 6. - Local density of states as a function of energy for two different interstices. $\varepsilon_{a}$ (denoted by the small circle on the abscissa) $=0.1$ and 0.3 . $W_{\mathrm{h}}$ denotes the half-bandwidth of the host metal. 
where

$g_{a a}(\varepsilon)=\left\{\begin{array}{r}{\left[\varepsilon-\varepsilon_{\mathrm{a}}-2 V_{a}^{2}\left(G_{0}+G_{2}\right)-i s\right]^{-1},} \\ \text { for the octahedral site } \\ {\left[\varepsilon-\varepsilon_{\mathrm{a}}-4 V_{a}^{2}\left(G_{0}+2 G_{111}+G_{2}\right)-i s\right]^{-1}} \\ \text { for the tetrahedral site. }\end{array}\right.$

The local density of states curve in figure 6 also demonstrates the difference in the electronic state associated with each interstice, and indicates the correspondence with the behaviour of the $\Delta W_{\mathrm{s}}$ curve ; except for the region near the lower band edge, the magnitude of $\rho_{a}(\varepsilon)$ for the tetrahedral site is greater than that for the octahedral site as far as the lower half of the virtual level (bonding state) is concerned. Therefore, the assumption of the strain energy model by Beshers [29] would probably not be valid for the parameter range of our calculations $\left({ }^{3}\right)$.

3.2 PAIR INTERACTION ENERGY IN THE b.c.c. LATTICE. - This section is mainly concerned with the interstitial solute atoms located at the tetrahedral interstices with particular emphasis on $\mathrm{H}$ in $\mathrm{Nb}$. In $\mathrm{Ta}$ or $\mathrm{Nb}$ the hydrogen occupies the tetrahedral interstices. In $\mathrm{Nb}-\mathrm{H}_{x}$, for example, one finds a gasliquid $\left(\alpha-\alpha^{\prime}\right)$ and a liquid-solid $\left(\alpha^{\prime}-\beta\right)$ phase transition [30]. The importance of the solute-solute (elastic) interaction for the $\alpha-\alpha^{\prime}$ phase transition has been stressed by Alefeld [31]. Now let us investigate the electronic contribution to this interaction. By the analogous calculation to the case of the single solute atom, we have $\operatorname{det}\left(1-G^{0} . V\right)$ as

$$
\begin{aligned}
\operatorname{det}\left(1-G^{0} V\right)=\left\{1-4 V_{a}^{2}\left(G_{0}+\right.\right. & \left.\left.2 G_{111}+G_{2}\right) G_{a a}\right\} \times \\
& \times\left\{1-4 V_{b}^{2}\left(G_{0}+2 G_{111}+G_{2}\right) G_{b b}\right\}-V_{a}^{2} V_{b}^{2} G_{a a} G_{b b} G_{s}^{2},
\end{aligned}
$$

where

$$
\begin{aligned}
G_{s}=G_{13}+G_{13^{\prime}}+G_{14}+G_{14^{\prime}}+G_{1^{\prime} 3}+G_{1^{\prime} 3^{\prime}}+G_{1^{\prime} 4}+ \\
+G_{1^{\prime} 4^{\prime}}+G_{23}+G_{23^{\prime}}+G_{24}+G_{24^{\prime}}+G_{2^{\prime} 3}+G_{2^{\prime} 3^{\prime}}+G_{2^{\prime} 4}+G_{2^{\prime} 4^{\prime}} .
\end{aligned}
$$

Thus, for the case of the nearest-neighbour pair interaction, det $\left(1-G^{0} . V\right)$ is written as

$$
\operatorname{det}\left(1-G^{0} \cdot V\right)=\left\{1-4 V_{a}^{2}\left(G_{0}+2 G_{111}+G_{2}\right) G_{a a}\right\}^{2}-V_{a}^{4} G_{a a}^{2}\left(3 G_{0}+8 G_{111}+4 G_{2}+G_{220}\right)^{2},
$$

where

$$
G_{220}=\frac{16}{\pi^{2}}\left[\left(2-k^{2}\right) K(k)-2 E(k)\right] \cdot\left[2 E(k)-\left(1-k^{2}\right) K(k)\right] .
$$

Since (3.13) is applicable to any nearest-neighbour pair, $\Delta W_{\mathrm{p}}^{\mathrm{T}}$ does not depend on the relative (or angular) position of the pair (in the vectorial sense). This would also be expected when dealing with the concentrated interstitial solid solutions. From (3.13) we obtain the pair interaction energy in the decomposed form as

$$
\begin{aligned}
& \Delta W_{\mathrm{p}}^{\mathrm{T}}=-\frac{2}{\pi} \int_{-\infty}^{\varepsilon_{\mathrm{f}}}\left[\arg \left\{\varepsilon-\varepsilon_{a}-4 V_{a}^{2}\left(G_{0}+2 G_{111}+G_{2}+G_{s} / 4\right)-i s\right\}+\right. \\
& \left.\quad+\arg \left\{\varepsilon-\varepsilon_{a}-4 V_{a}^{2}\left(G_{0}+2 G_{111}+G_{2}-G_{s} / 4\right)-i s\right\}-2 \arg \left\{\varepsilon-\varepsilon_{a}-4 V_{a}^{2}\left(G_{0}+2 G_{111}+G_{2}\right)-i s\right\}\right] \mathrm{d} \varepsilon .
\end{aligned}
$$

Here, we do not take into account the change in $\varepsilon_{a}$ due to the pair interaction, since the contribution of this effect to $\Delta W_{\mathrm{p}}^{\mathrm{T}}$ is expected to be cancelled out by the electron-electron interaction term to lowest order. The results of the numerical calculations of $\Delta W_{\mathrm{p}}^{\mathbf{T}}$ are shwon in figure 7 for $V_{a}=0.2, \varepsilon_{a}=0.1,0.3$ and 0.5 . The total screening charge (single interstitial) $\Delta Z$ and the occupation number $\left\langle n_{a}\right\rangle\left({ }^{4}\right)$ of the interstitial atom for $\varepsilon_{\mathrm{f}}=-0.04$ (representing $\mathrm{Nb}$ )

$\left({ }^{3}\right)$ In reference [29], the difference between the electronic energies associated with each interstice is not taken into account.

$\left({ }^{4}\right)$ Here, $\left\langle n_{a}\right\rangle=2\left\langle n_{a \sigma}\right\rangle=2\left\langle n_{a-\sigma}\right\rangle$. are as follows [47]; $\Delta Z=1.39,\left\langle n_{a}\right\rangle=0.94$ for $\varepsilon_{a}=0.1, \Delta Z=1.17,\left\langle n_{a}\right\rangle=0.60$ for $\varepsilon_{a}=0.3$ and $\Delta Z=0.92,\left\langle n_{a}\right\rangle=0.32$ for $\varepsilon_{a}=0.5$. Since the general features of the interaction curve are relatively insensitive to variations of $V_{a}$, the situation which represents $\mathrm{H}$ atoms in $\mathrm{Nb}$ might be included in our calculations. We see that figure 7 predicts the attractive interaction for the nearest-neighbour pair of $\mathrm{H}$ atoms in $\mathrm{Nb}$. In order to see this tendency more generally, we take into account here the direct interaction (hopping) between the interstitial atoms. In the case of the pair interactions, the inclusion of the direct interaction term is more complicated, but not more difficult. To take into account this direct interaction, 
we simply put the elements representing the direct After some tedious calculations, we get det $\left(1-G^{0} \cdot V\right)$ hopping, $T_{a b}$ and $T_{b a}$, into the potential matrix $V$. as

$$
\begin{aligned}
\operatorname{det}\left(1-G^{0} \cdot V\right) & =\left\{1-4 V_{a}^{2}\left(G_{0}+2 G_{111}+G_{2}\right) G_{a a}\right\} \times \\
\times & \left\{1-4 V_{b}^{2}\left(G_{0}+2 G_{111}+G_{2}\right) G_{b b}\right\}-G_{a a} G_{b b}\left(T_{a b}+V_{a} V_{b} . G_{s}\right)\left(T_{b a}+V_{b} V_{a} \cdot G_{s}\right) .
\end{aligned}
$$

In the case where $\left|4 V_{a}^{2}\left(G_{0}+2 G_{111}+G_{2}\right)\right| \gg\left|T_{a b}+V_{a}^{2} G_{s}\right|$, the pair interaction energy is written as

$$
\begin{aligned}
\Delta W_{\mathrm{p}}^{\mathrm{T}}=-\frac{2}{\pi} \int_{-\infty}^{\varepsilon_{\mathrm{f}}} & {\left[\arg \left\{\varepsilon-\varepsilon_{a}-4 V_{a}^{2}\left(G_{0}+2 G_{111}+G_{2}+G_{s} / 4-t / 4 V_{a}^{2}\right)-i s\right\}+\right.} \\
& \left.+\arg \left\{\varepsilon-\varepsilon_{a}-4 V_{a}^{2}\left(G_{0}+2 G_{111}+G_{2}-G_{s} / 4+t / 4 V_{a}^{2}\right)-i s\right\}\right] \mathrm{d} \varepsilon-2 \Delta W_{\mathrm{s}}^{\mathrm{T}},
\end{aligned}
$$

since we could expect the same cancellation as that mentioned before. Here $t=\left|T_{a b}\right|=\left|T_{b a}\right|$. For the numerical calculations, the magnitude of the direct hopping parameter $t$ is taken as, $t \leqq 0.1$ (in view of the nearest-neighbour $\mathrm{H}-\mathrm{H}$ distance in $\mathrm{Nb}(2.33 \AA)$, this value (0.1) might be too large; $\mathrm{H}-\mathrm{H}$ distance of the $\mathrm{H}_{2}$ molecule is $0.74 \AA$ and its binding energy is $4.74 \mathrm{eV})$. The results of the numerical calculations are given in figure 8 for $V_{a}=0.2, \varepsilon_{a}=0.3$ and $t=0 \sim 0.1$. The maximum value of $\left|\Delta W_{\mathrm{p}}^{\mathrm{T}}\right|$ is about $0.5 \mathrm{eV}$ for $W_{\mathrm{b}}=9.0 \mathrm{eV}(\mathrm{Nb})$ and generally indicates the importance of the electronic contribution to the pair interaction energy. We observe that the general feature of the pair interaction energy does not change drastically even if the direct interaction effect is taken into account. This is because the hopping potential $t$ used here is rather small compared with that of the

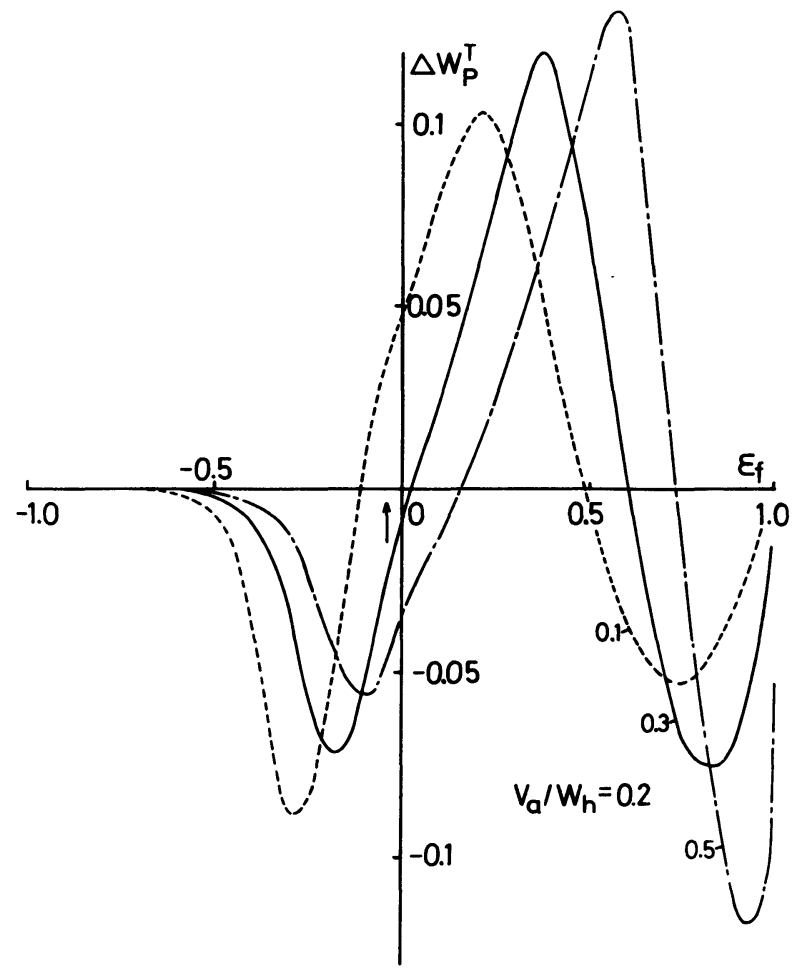

FIG. 7. - Nearest-neighbour pair-interaction energy between interstitials located at the tetrahedral sites; $\varepsilon_{a}=0.1,0.3$ and 0.5 . The Fermi level of $\mathrm{Nb}$ is indicated by the arrow.

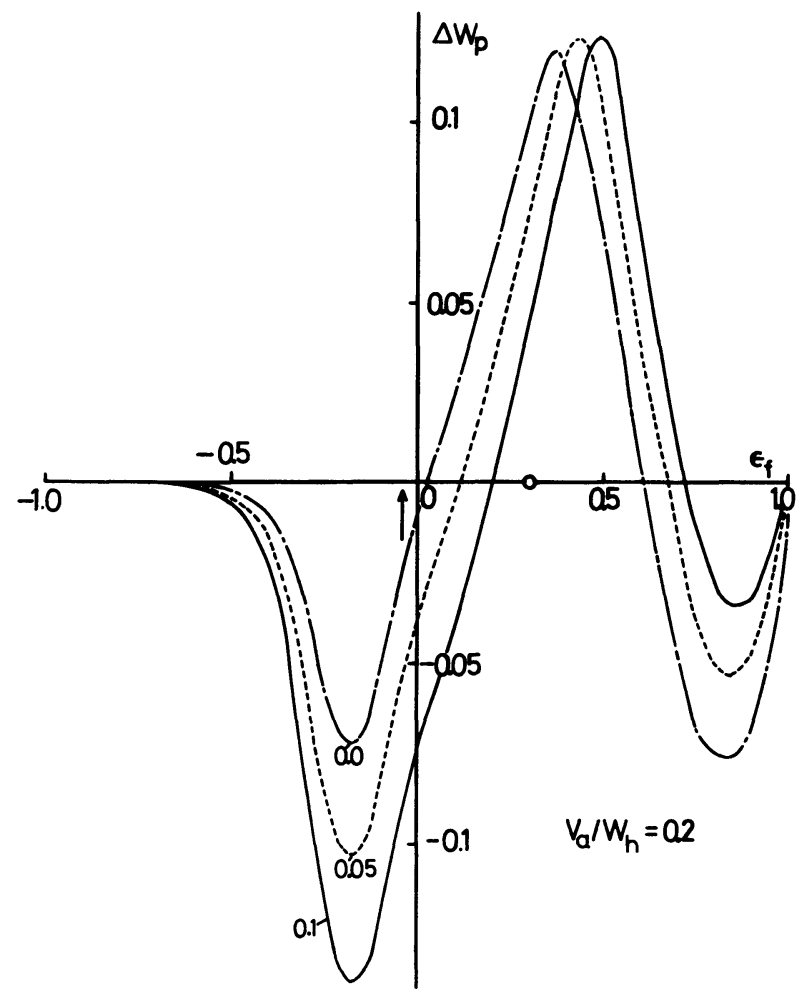

FIG. 8. - Nearest-neighbour pair-interaction energy between interstitials located at the tetrahedral sites. $\varepsilon_{a}$, denoted by the small circle on the abscissa, is 0.3 . $t$ (direct hopping parameter) $=0.0$, 0.05 , and 0.1 .

host metal. For the case of $V_{a}=0.2, \varepsilon_{a}=0.3$ and $\varepsilon_{\mathrm{f}}=-0.04$ (roughly representing the hydrogen atom in $\mathrm{Nb}$ ), the attractive solute-solute interaction energy increases if the direct interaction is included; from $-0.1 \mathrm{eV}(t=0)$ to $-0.23 \mathrm{eV}(t=0.05)$.

4. Interaction between interstitial and substitutional solute atoms. - In this section, we show that our general formalism also allows to investigate the interaction between the interstitial and substitutional solute atoms. The experimental studies to obtain information about the behavior of the IS interaction have widely been done recently, since the knowledge of the effect of alloying elements on the interstitial activity is of major importance for the understanding 
of phase equilibria and phase transformations in alloyed steels. From measurements of interstitial (carbon or nitrogen) activity in austenite [33, 34], it is clear that the carbon or nitrogen activity is decreased by the addition of chromium and manganese or vanadium as substitutional atoms and increased by cobalt or nickel. Alex and McLellan [5] applied a quasi-chemical approach to these ternary systems and obtained the attractive interactions for $\mathrm{Mn}-\mathrm{C}$ and $\mathrm{Cr}-\mathrm{C}$ and repulsive interaction for $\mathrm{Co}-\mathrm{C}$ in austenite. We want to answer the question as to why the interaction of the former case is attractive but the interaction of the latter case is repulsive.
In order to investigate these I-S interactions theoretically on our general formalism developed previously, we use the simple diagonal model of Koster and Slater (which has a single narrow band, in which the perturbation introduced by the solute atom is localized on a given lattice site) [35] for the substitutional atom. Let us denote the lattice site and the diagonal potential matrix element of the substitutional solute atom by $R_{3}$ and $V_{0}$, respectively. The notation of parameters concerning the interstitial solute atom is identical with that of the preceding sections. Following our general method, we obtain det $\left(1-G^{0} . V\right)$ as

$$
\operatorname{det}\left(1-G^{0} \cdot V\right)=1-V_{a}^{2} V_{0}\left(G_{13}+G_{23}\right)^{2} /\left[\left(1-G_{0} V_{0}\right)\left\{\varepsilon-\varepsilon_{a}-2 V_{a}^{2}\left(G_{0}+G_{1}\right)-i s\right\}\right],
$$

for the interstitial solute atoms in the bridge-binding interstices of the simple cubic lattice as well as in the octahedral interstices of b.c.c. lattice (in this case, $G_{1}$ is replaced by $G_{2}$ ). The nearest neighbour lattice sites of the interstitial atom are denoted by $R_{1}$ and $R_{2}$. For the tetrahedral interstices in the b.c.c. lattice, we have

$$
\begin{aligned}
\operatorname{det}(1 & \left.-G^{0} V\right)= \\
& =1-V_{a}^{2} V_{0}\left(G_{13}+G_{1^{\prime} 3}+G_{23}+G_{2^{\prime} 3}\right)^{2} /\left[\left(1-G_{0} V_{0}\right)\left\{\varepsilon-\varepsilon_{a}-4 V_{a}^{2}\left(G_{0}+2 G_{111}+G_{2}\right)-i s\right\}\right],
\end{aligned}
$$

where 1, 1', 2 and $2^{\prime}$ denote the nearest-neighbour lattice sites of the interstitial solute atom. For the octahedral interstices in the f.c.c. lattice,

$$
\begin{aligned}
\operatorname{det}\left(1-G^{0} V\right)=1-V_{a}^{2} V_{0}\left(G_{14}+G_{1^{\prime} 4}+G_{24}+\right. & \left.G_{2^{\prime} 4}+G_{34}+G_{3^{\prime} 4}\right)^{2} /\left[\left(1-G_{0} V_{0}\right) \times\right. \\
& \left.\times\left\{\varepsilon-\varepsilon_{a}-6 V_{a}^{2}\left(G_{0}+4 G_{110}+G_{2}\right)-i s\right\}\right] \\
& \{
\end{aligned}
$$

where $1,1^{\prime}, 2,2^{\prime}, 3$ and $3^{\prime}$ denote the nearest-neighbour lattice sites of the interstitial atom and 4 denotes the substitutional atom site. Thus we can get the IS interaction energy $\Delta W_{\text {IS }}$ as

$$
\begin{aligned}
\Delta W_{\mathrm{IS}} & =-\frac{2}{\pi} \int_{-\infty}^{\varepsilon_{\mathrm{f}}} \operatorname{Im} \ln \left(1-V_{a}^{2} V_{0}\left(G_{13}+G_{23}\right)^{2} /\left[\left(1-G_{0} V_{0}\right)\left\{\varepsilon-\varepsilon_{a}-2 V_{a}^{2}\left(G_{0}+G_{1}\right)-i s\right\}\right]\right) \mathrm{d} \varepsilon \\
& \cong \frac{2}{\pi} \int_{-\infty}^{\varepsilon_{\mathrm{f}}} \operatorname{Im} V_{a}^{2} V_{0}\left(G_{13}+G_{23}\right)^{2} /\left[\left(1-G_{0} V_{0}\right)\left\{\varepsilon-\varepsilon_{a}-2 V_{a}^{2}\left(G_{0}+G_{1}\right)-i s\right\}\right] \mathrm{d} \varepsilon
\end{aligned}
$$

for the interstitial solute atoms in the bridge-binding interstices of the simple cubic lattice and the octahedral interstices of the b.c.c. lattice $\left(G_{1} \rightarrow G_{2}\right)$. Here, the contributions coming from the changes in $\varepsilon_{a}$ and $V_{0}$ to $\Delta W_{\text {Is }}$ are cancelled out by those of the electron-electron interaction terms, to lowest order. Under the Hartree or Hartree-Fock approximation, such cancellation occurs generally. Similar expressions are obtained for the tetrahedral interstices of the b.c.c. lattice and for the octahedral interstices of the f.c.c. lattice. The asymptotic expression of the interaction energy in (4.4) is analogous to the first-order expression derived by Masuda for the vacancy and the impurity atom in noble metals [36]. In order to investigate the interaction between the simple interstitial atom (C or $\mathrm{N}$ ) and the substitutional solute elements centered around $\mathrm{Fe}$ in austenite qualitatively, we have carried out the numerical calculations for the simple cubic lattice under the conditions $\varepsilon_{a} \leqq 0$, $V_{a} / 2 T=1 \sim 2$ and $V_{0}=0.6448,0.3237,-0.4039$ and -1.0661 . The potential parameter $V_{0}$ is determined such that the total screening charge per spin, $\Delta Z$, becomes $-2 / 10,-1 / 10,1 / 10$ and $2 / 10$ for $\varepsilon_{\mathrm{f}}=0.7$, representing the $\mathrm{Cr}, \mathrm{Mn}$, Co and $\mathrm{Ni}$ impurity in $\mathrm{Fe}$ (paramagnetic). Since the single site approximation for the substitutional impurity potential is probably not valid for the system with bound states (FeCu...) we restrict the calculation of $\Delta W_{\text {IS }}$ to systems without bound states. The results of the numerical calculations are given in figure 9, and indicate the difference in the general behaviours of $\Delta W_{\mathrm{IS}}$, depending on the sign of $V_{0}$. This property is a consequence of the diagonal model of Koster and Slater and can be mathematically shown to be true when $V_{0}$ is sufficiently small. Since this fact is of such importance to interpret the experimental results that we will present 


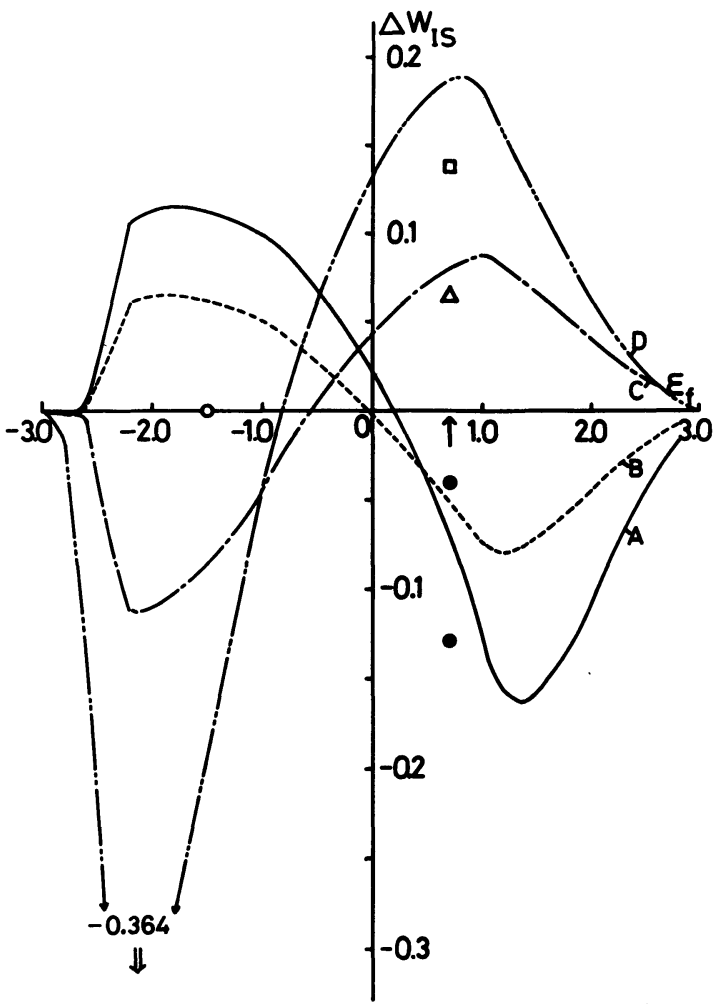

FIG. 9. - Nearest-neighbour IS interaction energy; $\varepsilon_{a}=-1.5$. The Fermi level of $\mathrm{Fe}$ (paramagnetic) is indicated by the arrow. $\square, \Delta, \odot$, and indicate the experimental values of the interaction energy between the carbon atom and $\mathrm{Ni}, \mathrm{Co}, \mathrm{Mn}$ and $\mathrm{Cr}$, respectively (from refs. [5], [33] and [34]). Nitrogen has almost the same character as carbon.

here the explicit proof of the above explanation. The integrand of (4.4) is decomposed as

$$
\begin{aligned}
& \left\{V_{0} /\left(1-G_{0} V_{0}\right)\right\} \times \\
& \quad \times\left[V_{a}^{2}\left(G_{13}+G_{23}\right)^{2} /\left\{\varepsilon-\varepsilon_{a}-2 V_{a}^{2}\left(G_{0}+G_{1}\right)-i s\right\}\right]
\end{aligned}
$$

and the imaginary part of the integrand is the sum of

$$
\begin{aligned}
& \operatorname{Im}\left\{V_{0} /\left(1-G_{0} V_{0}\right)\right\} \times \\
& \quad \times \operatorname{Re}\left[V_{a}^{2}\left(G_{13}+G_{23}\right)^{2} /\left\{\varepsilon-\varepsilon_{a}-2 V_{a}^{2}\left(G_{0}+G_{1}\right)-i s\right\}\right]
\end{aligned}
$$

and

$$
\begin{aligned}
& \operatorname{Re}\left\{V_{0} /\left(1-G_{0} V_{0}\right)\right\} \times \\
& \times \operatorname{Im}\left[V_{a}^{2}\left(G_{13}+G_{23}\right)^{2} /\left\{\varepsilon-\varepsilon_{a}-2 V_{a}^{2}\left(G_{0}+G_{1}\right)-i s\right\}\right] .
\end{aligned}
$$

Since

$$
\operatorname{Im}\left\{V_{0} /\left(1-G_{0} V_{0}\right)\right\}=V_{0}^{2} G_{0}^{\mathrm{I}} / D
$$

and

$$
\operatorname{Re}\left\{V_{0} /\left(1-G_{0} V_{0}\right)\right\}=V_{0}\left(1-G_{0}^{\mathrm{R}} V_{0}\right) / D,
$$

with

$$
D=\left(1-G_{0}^{\mathrm{R}} V_{0}\right)^{2}+\left(G_{0}^{\mathrm{I}} V_{0}\right)^{2},
$$

we see that the imaginary part of the integrand is, to first order, proportional to $V_{0}$. This discussion is capsulized in figure 9, which shows the effects of the sign of $V_{0}$ : we see that the sign of the I-S interaction energy curves of $\mathrm{A}$ and $\mathrm{B}$ is contrary to that of $\mathrm{C}$ and $\mathrm{D}$ for the most part of the band range. Although there is a violent oscillation near the lower band edge (precursor state of the bound state) for $V_{0}=-1.0661$, we observe that $\Delta W_{\mathrm{IS}}$ varies in a rather predictable fashion, depending on the sign of $V_{0}: \Delta W_{\text {IS }}$ varies as repulsive $\rightarrow$ attractive for positive $V_{0}$ and attractive $\rightarrow$ repulsive for negative $V_{0}$. Most importantly (and surprisingly enough), our simple calculation could explain the experimental observation for $\mathrm{Fe}$ based ternary alloys containing simple gas atoms such as $\mathrm{N}$ or $\mathrm{C}$; for the interstitial solute atoms with $\varepsilon_{a} \leqq-0.5$ (and not so below the lower band edge), the substitutional solute atoms such as $\mathrm{Cr}$ or $\mathrm{Mn}$ interact attractively with them in $\mathrm{Fe}$ (paramagnetic), while $\mathrm{Co}$ or $\mathrm{Ni}$ interacts repulsively with them. The range of $\varepsilon_{a}$ considered here for $\mathrm{C}$ or $\mathrm{N}$ is reasonable in view of the parameters concerned, such as the first ionization energy of the carbon or nitrogen atom $(11.26 \sim 14.53 \mathrm{eV}$ [37]). Therefore, we may conclude that our simple model for the IS interaction could give a qualitative description of the ternary Fe-M-C or $\mathrm{Fe}-\mathrm{M}-\mathrm{N}$ austenite, where $\mathrm{M}$ denotes $\mathrm{Cr}, \mathrm{Mn}$, Co and $\mathrm{Ni}$.

5. Discussion and conclusion. - In the previous sections, we have investigated the problems of both the I-I and I-S interaction within the context of the tight-binding approximation. Although the present theoretical method closely parallels that developed by Einstein and Schrieffer for the adatom interaction, it further allows to investigate the interaction between the interstitial and substitutional solute atoms. Furthermore, we have added the direct interaction between the nearest-neighbour interstitial atoms to the indirect interaction energy (Einstein and Schrieffer did not calculate this interaction in their work). These developments are of considerable importance since the systems considered here have only been discussed by the elastic theory or other phenomenological theory such as the quasi-chemical approach. Nevertheless, the present model is sufficiently crude that the calculations are primarily of qualitative significance, i.e. it is not possible to this stage to draw comparison with specific systems.

The I-I interaction energies are calculated for both the simple cubic and body centered cubic tightbinding solids with parameters $W_{\mathrm{b}}, V_{a}, \varepsilon_{\mathrm{f}}$ and $\varepsilon_{a}$ ( $W_{\mathrm{b}}$ and $\varepsilon_{\mathrm{f}}$ are fixed from data on the host metals). The hopping potentials $V_{a}$ are estimated by the simple approximation of a Gaussian form of atomic orbitals and a screened Coulomb form of the impurity potential (interstitial atom). The calculated $V_{a}$ are in the range $1.0 \sim 2.0 \mathrm{eV}$, the same order as those for chemisorbed gas atoms on the transition metal 
surfaces. Since the I-I interaction energy curves in the interior of the band merely increase in amplitude with increasing potential and with little change in shape, the uncertainty in calculating $V_{a}$ is not serious as far as the qualitative properties are concerned. $\Delta W_{\mathrm{p}}$ is then determined uniquely as a function of the positions of $\varepsilon_{a}$ for the single-solute atom adsorption. For the same $\varepsilon_{a}$, the nearest-neighbour pair interaction energies in the b.c.c. lattice (tetrahedral sites) have almost the same character as those in the simple cubic lattice (bridge-binding site) : the general feature of the nearest-neighbour pair interaction energy mainly depends on the band filling and the position of $\varepsilon_{a}$. In particular, we have investigated the I-I interaction, with emphasis on the $\mathrm{H}-\mathrm{H}$ pair interaction in $\mathrm{Nb}$. Although we could not calculate this interaction energy quantitatively, the qualitative aspects of this interaction have been investigated in detail : for the case of $V_{a}=0.2, \varepsilon_{a}=0.3$ and $\varepsilon_{\mathrm{f}}=-0.04$ (roughly representing the hydrogen atom in $\mathrm{Nb}$ ), the attractive solute-solute $(\mathrm{H}-\mathrm{H})$ interaction energy is obtained $(\approx-0.1 \mathrm{eV})$. The direct interaction also enlarges this tendency $(-0.2 \mathrm{eV}$ for $t=0.05)$. Therefore, the assumption that the dominant attractive interaction between the hydrogen atoms in $\mathrm{Nb}$ is of an elastic nature [31] is very questionable. Furthermore, the magnitude of the pair interaction energy $\Delta W_{\mathrm{p}}^{\mathrm{T}}$ is in the range of $\left|\Delta W_{\mathrm{p}}^{\mathrm{T}}\right| \lesssim 0.11(0.5 \mathrm{eV})$ for $V_{a}=0.2$. This fact clearly demonstrates the importance of the electronic contribution to the interstitial interaction energies, such as the $\mathrm{H}-\mathrm{H}$ interaction in $\mathrm{Nb}$. Thus, in discussing the $\alpha-\alpha^{\prime}$ phase transition in the $\mathrm{Nb}-\mathrm{H}$ system, the electronic interaction between the hydrogen atoms should be included. In this respect, it is necessary to reexamine the discussion of the $\alpha-\alpha^{\prime}$ phase transition (interstitial condensation) based on the elastic theory. Since the $\alpha-\alpha^{\prime}$ phase transition of the $\mathrm{Nb}-\mathrm{H}$ system is of interest in some respects and has been discussed by using the elastic theory by several authors [31], we briefly discuss the elastic energy based on the continuum model. Alefeld et al. [31] claimed to have shown that interstitial solute atoms interact attractively on the base of the elastic energy and, thus, they tend to condense at low temperatures. However, we think that such a conclusion is valid only under particular conditions, as shown in the following.

Let an interstitial solute atom be characterized by the uniform (stress-free) transformation strain $e_{i j}^{\mathrm{T}}$ in a domain with the volume $\tilde{V}_{0}$. First we consider the interaction between two solute atoms. The elastic interaction energy, $E_{\mathrm{l}}$, between the two solute atoms is given by

$E_{\mathrm{I}}=-e_{i j}^{\mathrm{T}} \int_{\tilde{V}_{b}} \sigma_{i j}(a) \mathrm{d} v=-e_{i j}^{\mathrm{T}} \int_{\tilde{V}_{a}} \sigma_{i j}(b) \mathrm{d} v$,

where $\sigma_{i j}(a)$ and $\sigma_{i j}(b)$ are the stresses due to solute atoms $a$ (volume $\tilde{V}_{a}$ ) and $b\left(\tilde{V}_{b}\right)$ and the summation convention is used [38-39]. When only the interaction between the two solute atoms is considered, the stresses, $\sigma_{i j}(a)$ and $\sigma_{i j}(b)$ can be computed by assuming them to be present in a infinite medium, except for the situations where both the solute atoms are situated very close to the free surface of the actual finite specimen. It has been shown that if a domain $\tilde{V}_{0}$ with transformation strain $e_{i j}^{\mathrm{T}}$ produces a stress field $\sigma_{i j}^{\infty}$ in an infinite medium,

$$
\int_{\tilde{V}_{\mathbf{B}}-\tilde{V}_{\mathbf{A}}} \sigma_{i j}^{\infty} \mathrm{d} v=0
$$

when $\tilde{V}_{\mathbf{B}} \supset \tilde{V}_{\mathrm{A}} \supset \tilde{V}_{0}$ and $\tilde{V}_{\mathrm{A}}$ and $\tilde{V}_{\mathrm{B}}$ are the ellipsoidal domain with the similar shape [40]. This indicates that depending on the relative position (in a vectorial sense) of solute atoms $a$ and $b$, the elastic interaction energy can be positive or negative. Of course, since the magnitude of $\sigma_{i j}^{\infty}$ decreases as the inverse of the cube of the distance from the center of the solute atom [41], the closer the separation of the two solute atoms, the smaller the interaction energy, if they are situated in a particular angular position and the transformation strain has particular values. In order to claim that there is an attractive interaction between the solute atoms, we have to specify the angular position which depends on $e_{i j}^{\mathrm{T}}$. We would like to point out here that the nearest-neighbour interaction energy (electronic) $\Delta W_{\mathrm{p}}^{\mathrm{T}}$ for $\mathrm{H}-\mathrm{H}$ in $\mathrm{Nb}$ tends to become attractive and it does not depend on the relative (angular) position of the pair.

Next we discuss the case where a large number of solute atoms in a finite specimen is condensed. Before condensation, the average elastic energy, $E_{\mathrm{el}}$, per unit volume is given by [42]

with

$$
E_{\mathrm{el}}=-\frac{1}{2} f(1-f) \sigma_{i j}^{\infty} e_{i j}^{\mathrm{T}},
$$

$$
\sigma_{i j}^{\infty}=C_{i j k l}\left\{S_{k l m n}\left(\tilde{V}_{0}\right) e_{m n}^{\mathrm{T}}-e_{k l}^{\mathrm{T}}\right\},
$$

where $f$ is the volume fraction of the solute atoms (representing the concentration), the shape of the solute atom is assumed ellipsoidal and $S_{k l m n}\left(\widetilde{V}_{0}\right)$ is the Eshelby tensor [41] appropriate for the ellipsoidal domain $\tilde{V}_{0}$ aidd $C_{i j k l}$ the elastic constant. For simplicity, we treat the case where the solute atoms are enriched, in a random manner, in domains $\tilde{V}$ of an ellipsoidal shape and such domains are distributed randomly and occupy the volume fraction $f_{\mathrm{d}}$. When a single domain is taken out from the specimen, the internal stress in this domain is, on the average, given by [42]

$$
\text { and } \left.\begin{array}{ll}
\left\langle\sigma_{i j}\right\rangle_{\mathrm{M}} & =-f_{1} \sigma_{i j}^{\infty} \quad \text { in matrix of } \tilde{V} \\
\left\langle\sigma_{i j}\right\rangle_{\tilde{V}_{0}}=\left(1-f_{1}\right) \sigma_{i j}^{\infty} & \text { in a solute atom in } \tilde{V}
\end{array}\right\} .
$$

Here, $f_{1}$ is the volume fraction of the solute atoms within $\tilde{V}\left(f_{1} f_{\mathrm{d}}=f\right)$. The domain $\tilde{V}$ itself has now 
the transformation strain $f_{1}$. When this domain is replaced back to the hole from which it has been taken out, a new internal stress is developed because of $f_{1} e_{i j}^{\mathrm{T}}$. Since there are many and randomly distributed similar domains in the finite specimen, the new additional internal stress within the domain $\tilde{V}$ is, on the average,

$$
\left\langle\sigma_{i j}\right\rangle_{\widetilde{V}}=\left(1-f_{\mathrm{d}}\right) \sigma_{i j}^{\infty}
$$

with

$$
\sigma_{i j}^{\infty}=C_{i j k l}\left\{S_{k l m n}(\tilde{V}) f_{1} e_{m n}^{\mathrm{T}}-f_{1} e_{k l}^{\mathrm{T}}\right\},
$$

where $S_{k l m n}(\tilde{V})$ is the Eshelby tensor appropriate for the enriched ellipsoidal domain [42]. Thus, the total internal stress within a solute atom in the finite specimen is

$$
\sigma_{i j}=\left(1-f_{1}\right) \cdot \sigma_{i j}^{\infty}+\left(1-f_{\mathrm{d}}\right) \cdot \sigma_{i j}^{\infty} .
$$

From this, the elastic energy, $E_{\mathrm{el}}^{\prime}$, per unit volume of the specimen after condensation is calculated as

$E_{\mathrm{el}}^{\prime}=-\frac{1}{2} f\left\{\left(1-f_{1}\right) \sigma_{i j}^{\infty}+\left(1-f_{\mathrm{d}}\right) \sigma_{i j}^{\infty}\right\} e_{i j}^{\mathrm{T}}$.

An interesting case is that in which the solute atom and the enriched domain have a similar ellipsoidal shape. In such a case, $E_{\mathrm{el}}^{\prime}$ is equal to $E_{\mathrm{el}}$ and there is no energy change upon condensation. However, when $e_{i j}^{\mathrm{T}}$ has a particular value and a solute atom and an enriched domain have particular shapes, $E_{\mathrm{el}}^{\prime}$ can be smaller than $E_{\mathrm{el}}$. For example, if $e_{33}^{\mathrm{T}}=\varepsilon$, other components of $e_{i j}^{T}=0$ and a solute atom is assumed to occupy a spherical region,

$$
E_{\mathrm{el}}=\frac{8 f(1-f) \mu}{15(1-v)} \varepsilon^{2} .
$$

Here the specimen is assumed elastically isotropic and $\mu$ and $v$ are the shear modulus and the Poissons ratio, respectively. When solute atoms are condensed in disc shaped domains $\left(x^{2} / a^{2}+y^{2} / a^{2}+z^{2} / c^{2}=1\right.$, $c \ll a), E_{\mathrm{el}}^{\prime}$ is calculated as

$$
E_{\mathrm{el}}^{\prime}=\frac{8 f\left(1-f_{1}\right) \mu}{15(1-v)} \varepsilon^{2} .
$$

Thus, upon condensation $\left(f_{1}>f\right), E_{\mathrm{el}}^{\prime}<E_{\mathrm{el}}$. Of course, there is an opposite case where $E_{\mathrm{el}}^{\prime}>E_{\mathrm{el}}$ (for example, the enriched region is described by $\left.\left(x^{2}+y^{2}\right) / a^{2}+z^{2} / c^{2}=1, c>a\right)$.

From the above discussions, it is clear that one can discuss the possibility of solute atom condensation, based on the elastic energy, only if the relative position of the solute atoms or, presumably equivalently, the shape of the enriched region is specified. Depending on these factors, the change in the elastic energy upon the decrease of the mutual distance of the solute atoms is negative or positive. These conclusions clearly deny the claim by Alefeld and Alefeld et al. [31] on the simple consideration. Although it seems unnecessary to criticize the argument by these authors, we would like to point out that they never showed the elastic energy as a function of the fluctuation of the solute atom concentration or the volume fraction and the solute concentration of the enriched regions. Only through such expressions, one can discuss the possibility of the solute condensation. Therefore, it seems to be still an open question whether the elastic interaction of hydrogen atoms in $\mathrm{Nb}$ can account for the $\alpha-\alpha^{\prime}$ (gas-liquid) phase transition : we have demonstrated the importance of the electronic interaction between interstitial solute atoms by estimating the magnitude of the pair interaction.

The I-S interaction energies are calculated for a reasonable range of parameters with particular emphasis on Fe (paramagnetic) base ternary alloys. The magnitude of the I-S interaction energy also indicates the importance of the electronic contribution. It is gratifying that our simple calculation can account for the experimental observations; 3-d transition elements located in the periodic system on the left of $\mathrm{Fe}$ interact with the simple gas atom interstitials (C or N) attractively, while the elements located on the right of $\mathrm{Fe}$ interact repulsively with them. Calculations also indicate that our scale of $V_{a}$ is reasonable. The present treatment of the I-S interaction can straightforwardly be extended to the case of the interstitial-vacancy pair [43]. For this purpose, however, it is necessary at least to take into account the diagonal matrix elements on $Z$ sites neighbouring the vacancy site [44].

Finally we note that the present approach is also applicable to the study of the magnetic properties of the interstitial solid solutions (within the HartreeFock approximation). Fujita et al. [9] are using Mössbauer spectra to investigate the internal magnetic field, isomer shift, and the electric quadrupole effect for the iron atom in the martensite of high carbon steel. Detailed calculations on this matter will be presented separately.

Appendix. - We derive the site diagonal Green's functions. $y_{1}$ and $y_{a u}$. lor the single solute case. We start with the resolvent form of the one-particle Green's function given by

$$
\begin{aligned}
g= & (\varepsilon-\mathcal{H}-i s)^{-1}= \\
& =\left(\varepsilon-H_{0}-i s\right)^{-1}+\left(\varepsilon-\mathcal{H}_{0}-i s\right)^{-1} \mathrm{Vg} .
\end{aligned}
$$

For the single solute in the tetrahedral site of the b.c.c. lattice, the matrix elements of the Green's function are written as

$$
\begin{aligned}
& g_{i i}=G_{i i}+\left(G_{i 1} V_{1 a}+G_{i 1^{\prime}} V_{1^{\prime} a}+G_{i 2} V_{2 a}+G_{i 2^{\prime}} V_{2^{\prime} a}\right) g_{a i}, \\
& g_{a i}=G_{a a}\left(V_{a 1} g_{1 i}+V_{a 1^{\prime}} g_{1^{\prime} i}+V_{a 2} g_{2 i}+V_{a 2^{\prime}} g_{2^{\prime} i}\right)
\end{aligned}
$$

$g_{a a}=G_{a a}\left(1+V_{a 1} g_{1 a}+V_{a 1^{\prime}} g_{1^{\prime} a}+V_{a 2} g_{2 a}+V_{a 2^{\prime}} g_{2^{\prime} a}\right), ~ 。$ 
where we have assumed that the interstitial orbital is orthogonal to all Wannier functions of the host d-band. Solving (A.2), (A.3) and (A.4), we have

$$
\begin{aligned}
& g_{a a}=\left[\varepsilon-\varepsilon_{a}-4 V_{a}^{2}\left(G_{0}+2 G_{111}+G_{2}\right)-i s\right]^{-1}, \\
& g_{i i}=G_{i i}+g_{a a} V_{a}^{2}\left(G_{1 i}+G_{1^{\prime} i}+G_{2 i}+G_{2^{\prime} i}\right)^{2} .
\end{aligned}
$$

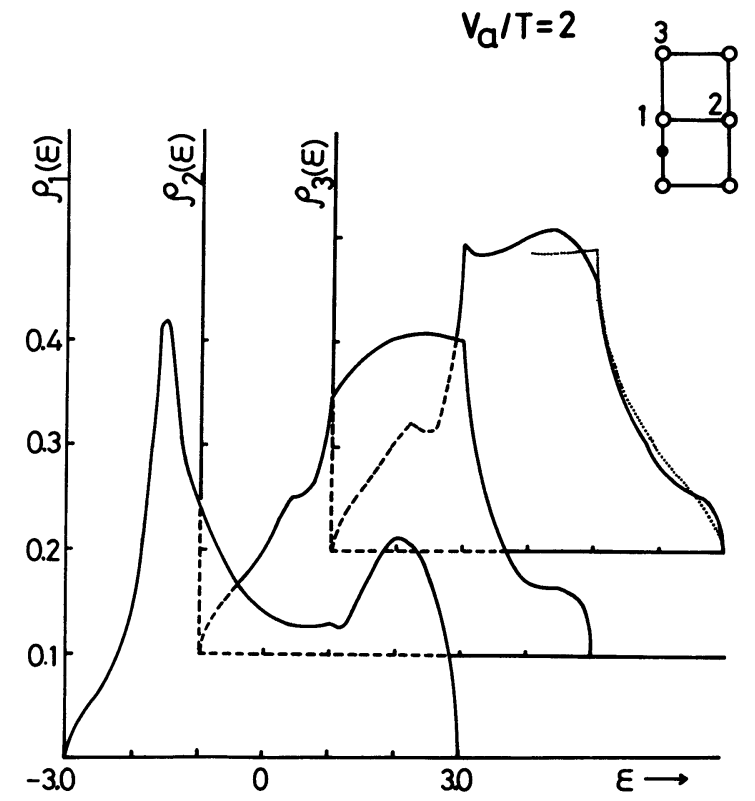

FIG. 10. - Local density of states for the host metal atoms neighbouring the interstitial solute atom. The dotted curve in the upper figure is the unperturbed d-band density of states per atom.
Here we do not treat Coulomb interaction effects $\left(U n_{a \uparrow} n_{a \downarrow}\right)$ explicitly; one must rescale our $\varepsilon_{a}$ by $U\left\langle n_{a}\right\rangle$ (Hartree-Fock approximation). Similar expressions are given for the bridge-binding interstice of the simple cubic lattice and the octahedral site of the b.c.c. lattice. The local density of states curves at the host metal sites are presented for the simple cubic lattice in figure 10 . We observe the drastic change in the density of states at the host metal sites; this clearly suggests that the rigid band (screened proton) model is not adequate for the description of the interstitial solid solutions.

The total screening charge, $\Delta Z$, is given from (A.5) and (A.6) as

$$
\Delta Z=\frac{1}{\pi} \operatorname{Im} \int_{-\infty}^{\varepsilon_{\mathrm{f}}}\left\{g_{a a}+\sum_{i}\left(g_{i i}-G_{i i}\right)\right\} \mathrm{d} \varepsilon .
$$

After a bit of algebra, we have

$$
\begin{aligned}
\Delta Z & =-\frac{1}{\pi} \tan ^{-1} \times \\
& \times \frac{4 V_{a}^{2} \operatorname{Im}\left\{G_{0}\left(\varepsilon_{\mathrm{f}}\right)+2 G_{111}\left(\varepsilon_{\mathrm{f}}\right)+G_{2}\left(\varepsilon_{\mathrm{f}}\right)\right\}}{\varepsilon_{\mathrm{f}}-\varepsilon_{a}-4 V_{a}^{2} \operatorname{Re}\left\{G_{0}\left(\varepsilon_{\mathrm{f}}\right)+2 G_{111}\left(\varepsilon_{\mathrm{f}}\right)+G_{2}\left(\varepsilon_{\mathrm{f}}\right)\right\}} .
\end{aligned}
$$

\section{References}

[1] Matsumoto, T., Sasaki, Y. and Hihara, M., J. Phys. \& Chem. Solids 36 (1975) 215.

[2] Fast, J. D. and Dijksta, L. J., Philips Tech. Rev. 13 (1951) 172 Dijksta, L. J. and Sladek, R. J., Trans. Am. Inst. Min. Metall. Pet. Eng. 197 (1953) 69.

Leak, D., Thomas, W. and Leak, G., Acta Metall. 3 (1955) 501. Ritchie, I. G. and Rawlings, R., Acta Metall. 15 (1967) 491.

[3] Hardy, J. R., J. Phys. \& Chem. Solids 15 (1960) 39 ; 29 (1968) 2009.

Tewary, V. K., J. Phys. F 3 (1973) 1275, 1515.

Bullough, R. and Tewary, V. K., Interatomic Potentials and Simulation of Lattice Defects (New York : Plenum) 1972, p. 155.

[4] Horner, H. and Wagner, H., J. Phys. C 7 (1974) 3305.

[5] Alex, K. and Mclellan, R. B., J. Phys. \& Chem. Solids 32 (1971) 449.

[6] Friedel, J., Phil. Mag. 43 (1952) 153, Ber. Bunsenges. Phys. Chem. 76 (1972) 828.

Ebisuzaki, Y. and O'Keefre, M., J. Phys. Chem. 72 (1968) 4695.

[7] Newns, D. M., Phys. Rev. 178 (1969) 1123.

GOMER, R. and SchriefFer, J. R., Surf. Sci. 25 (1971) 315.

SCHRIEFFer, J. R., J. Vac. Sci. Technol. 9 (1971) 561

Brenig, W. and Schonhammer, K.. Z. Phys. 267 (1974) 201.

Gadzuk, J. W., Surf. Sci. 43 (1974) 44 ; Phys. Rev. B 10 (1974) 5030.

Paulson, R. H. and Schriefrer, J. R., Surf. Sci. 48 (1975) 329.

[8] Einstein, T. L. and Schrieffer, J. R., Phys. Rev. B 7 (1973) 3629.

[9] Moriya, T., Ino, H., Fujita, F. E. and Maeda, Y., J. Phys Soc. Japan 24 (1968) 60.
Moriya, T., Sumitomo; Y., Ino, H., Fujita, F. E. and Maeda, Y., J. Phys. Soc. Japan 35 (1973) 1378.

[10] Gielen, P. M. and Kaplow, R., Acta Metall. 15 (1967) 49.

Ron, M., Kidron, A. and Schechter, H., J. Appl. Phys. 38 (1967) 590.

Genin, J. M. and Flinn, P. A., Trans. Am. Inst. Min. Metall. Pet. Eng. 242 (1968) 1419.

[11] Kim, T. K. and TaKahashi, M., Appl. Phys. Lett. 20 (1972) 492.

[12] Anderson, P. W., Phys. Rev. 124 (1961) 41.

[13] Friedel, J., The Physics of Metals (Cambridge University Press) 1969 , p. 340.

[14] Ducastelle, F., J. Physique 31 (1970) 1055.

[15] Lannoo, M. and Allan, G., J. Phys. \& Chem. Solids 32 (1971) 637.

[16] Kauffer, E. and Gerl, M., J. Physique 35 (1974) 839.

[17] Cyrot-Lackmann, F., J. Phys. \& Chem. Solids 29 (1968) 1235.

Allan, G. and Lenglart, P., Surf. Sci. 15 (1969) 101.

[18] Oriani, R. A. and Gonzalez, O. D., Trans. Am. Inst. Min. Metall. Pet. Eng. 239 (1967) 1041.

KnaAK, J. and EichenaueR, W., Z. Naturforsch. 23a (1968) 1783.

Einziger, R. E. and Huntington, H. B., J. Phys. \& Chem. Solids 35 (1974) 1563.

[19] Simons, J. W. and Flanagan, T. B., Can. J. Chem. 43 (1965) 1665.

Brodowsky, H., Z. Phys. Chem. 44 (1965) 129.

[20] BURCH, R., Trans. Faraday Soc. 66 (1970) 736.

[21] Kim, D. J. and NagaoKa, Y., Prog. Theor. Phys. 30 (1963) 743.

[22] Grimley, T. B. and WalKeR, S. M., Surf. Sci. 14 (1969) 395.

[23] Morita, T., J. Phys. A 8 (1975) 478.

[24] Horiguchi, T. and Morita, T., J. Phys. C 8 (1975) L 232.

[25] Allan, G., Ann. Phys. 5 (1970) 169. 
[26] Wolfram, T. and Callaway, J., Phys. Rev. 130 (1963) 2207. [27] Morita, T. and Horiguchi, T., Numer. Math. 20 (1973) 425. [28] Vykhodets, V. B., Gol'tsov, V. A. and Gel'D, P. V., Phys. Met. Metallogr. 25 (1968) 133.

[29] Beshers, D. N., J. Appl. Phys. 36 (1965) 290.

[30] Walter, R. J. and Chaudler, W. T., Trans. Am. Inst. Min. Metall. Pet. Eng. 233 (1965) 762.

[31] Alefeld, G., Phys. stat. sol. 32 (1969) 67; Ber. Bunsenges. Phys. Chem. 76 (1972) 746.

Conrad, H., Bauer, G., Alefeld, G., Springer, T. and Schmatz, W., Z. Phys. 266 (1974) 239.

[32] Joyce, G. S., J. Phys. C 1 (1971) 1510.

[33] Sмiтн, R. P., Trans. Am. Inst. Min. Metall. Pet. Eng. 70 (1948) 2724 ; 233 (1965) 397.

Flender, H. and WeVER, H., Arch. Eisenhuttenwes. 10 (1963) 727.

[34] Uhrenius, B., Scand. J. Metall. 2 (1973) 177.

[35] Koster, G. F. and Slater, J. C., Phys. Rev. 96 (1954) 1208.

[36] Masuda, K., Phys. Lett. 45A (1973) 381 ; J. Phys. F 5 (1975) 205.

[37] Moore, C. E., Atomic Energy Levels as Derived from the Analysis of Optical Spectra (Circular of the National Bureau of Standards, Washington D.C., 1949-1958. Vol. III).
[38] Eshelby, J. D., Solid State Phys. 3 (1956) 79.

[39] Mura, T., Adv. Mater. Res. 3 (1968) 1.

[40] Tanaka, K. and Mori, T., J. Elasticity 2 (1972) 199.

[41] Eshelby, J. D., Proc. R. Soc. A 241 (1957) 376.

[42] Mori, T. and TanaKa, K., Acta Metall. 21 (1973) 571.

[43] Damask, A. C. and Fujita, F. E., Acta Metall. 12 (1964) 331. Irmer, V. and Feller-KniePMeIER, M., Phil. Mag. 25 (1972) 1345.

Homan, C. G., Acta Metall. 12 (1964) 1071.

[44] Masuda, K., Phys. stat. sol. 62b (1974) 559.

Demangeat, C., Ann. Phys. (Paris) 8 (1973-4) 107.

[45] Gallagher, P. T. and Oates, W. A., Trans. Am. Inst. Min. Metall. Pet. Eng. 245 (1969) 179.

Flanagan, T. B. and OAtes, W. A., Ber. Bunsenges. Phys. Chem. 76 (1972) 706.

[46] Natta, M. and Toulouse, G., Phys. Lett. 24A (1967) 205. Parlebas, J. C., Demangeat, C. and Gautier, F., J. Phys. F 5 (1975) L 18

[47] Positive polarity of our calculation of $\left\langle n_{a}\right\rangle$ is in accord with the electro-transport or positron annihilation experiments (Abe, Y., Hasegawa, M., Suzuki, T., Asano, H. and Hirabayashi, M., Fall Meeting of the Japan Institute of Metals 1975). 